\title{
Exotic topological order in fractal spin liquids
}

\author{
Beni Yoshida \\ Institute for Quantum Information and Matter, California Institute of Technology, Pasadena, California 91125, USA
}

(Received 1 July 2013; published 12 September 2013)

\begin{abstract}
We present a large class of three-dimensional spin models that possess topological order with stability against local perturbations, but are beyond description of topological quantum field theory. Conventional topological spin liquids, on a formal level, may be viewed as condensation of stringlike extended objects with discrete gauge symmetries, being at fixed points with continuous scale symmetries. In contrast, ground states of fractal spin liquids are condensation of highly fluctuating fractal objects with certain algebraic symmetries, corresponding to limit cycles under real-space renormalization group transformations which naturally arise from discrete scale symmetries of underlying fractal geometries. A particular class of three-dimensional models proposed in this paper may potentially saturate quantum information storage capacity for local spin systems.
\end{abstract}

DOI: $10.1103 /$ PhysRevB.88.125122

PACS number(s): 03.67.-a, 05.30.- d, 05.50.+q

\section{INTRODUCTION}

In a quantum many-body system at zero temperature, topological order may arise when a gapped ground state possesses long-range entanglement that can not be detected by any local measurement or local order parameter. ${ }^{1,2}$ Its ground-state properties are stable against any types of local perturbations, regardless of symmetries of perturbations, and depend only on global properties of geometric manifold on which the whole system is supported. The discovery of topological order, such as fractional quantum Hall systems, ${ }^{3,4}$ came as a great surprise as they are beyond description of the Landau-Ginzburg theory which was once believed to be the ultimate theory of a quantum many-body system. It is now widely believed that the notion of topological order is essential in understanding the emergence of quantum phases with no local order in gapped quantum spin liquids, as seen in some frustrated antiferromagnets. ${ }^{5-12}$ The study of topologically ordered systems is also of practical importance as they are physically natural platforms for realizations of fault-tolerant quantum information processing. ${ }^{2}$

For topologically ordered spin liquids with discrete gauge symmetries, their low-energy behavior is relatively well understood on a formal level as they are effectively described by topological quantum field theory (TQFT), ${ }^{1,6}$ a field theory with invariance under continuous deformations (diffeomorphism). ${ }^{13,14}$ This is because their physical properties do not depend on local structures of systems, and depend only on topological properties of geometric manifolds. A fairly complete class of two-dimensional TQFT-based spin systems with nonchiral topological order has been proposed by Levin and Wen where condensation of highly fluctuating extended objects, called "string nets," are found to be responsible for emergence of topological order. ${ }^{15}$

Yet, in some cases, quantum spin liquids may exhibit topological order that is beyond description of TQFT. For example, in three spatial dimensions, the cubic code, recently proposed by Haah, ${ }^{16}$ possesses topological order with stability against local perturbations, but are completely different from conventional topological spin liquids. For one thing, the number of degenerate ground states is exponential in the linear length of the lattice. Furthermore, unlike string-net condensates, the model is free from stringlike extended objects, and the mobility of quasiparticle excitations is highly constrained via some algebraic rules. The discovery of the cubic code and relevant models ${ }^{17,18}$ clearly indicates that classification of topological phases via TQFT is incomplete; TQFT is just a subset of some universal theory of topological order which is yet to be found. The necessary first step is to find a family of topological spin liquids that are beyond TQFT.

The goal of this paper is to present a large class of exactly solvable topological spin liquids on a three-dimensional lattice which possess exotic topological order beyond TQFT. Instead of stringlike (one-dimensional) or membranelike (twodimensional) objects with continuous geometries, ground states are condensation of extended objects with noninteger dimensionality, namely, fractal objects. In this paper, we discuss physical properties of such quantum fractal liquids.

Emergence of fractal objects in correlated spin systems is not a completely new idea. Newman and Moore proposed a toy model of two-dimensional classical spin liquid with a large number of degenerate ground states whose spin configurations resemble the Sierpinski triangle. ${ }^{19}$ By generalizing their construction, we proved that a family of such fractal systems, referred to as classical fractal liquids in this paper, saturates a theoretical limit on classical information storage capacity of local Hamiltonians with mass gap. ${ }^{20}$ As demonstrated in this paper, ground states of classical fractal liquids do not have continuous scale symmetries, but have discrete scale symmetries only, exhibiting limit cycle behaviors under real-space RG transformations. Such exotic features of classical fractal liquids indicate a possibility of novel quantum phases beyond field theory with continuous scale invariance. Quantum fractal liquids can be viewed as natural generalization of classical fractal liquids to a quantum setting, and may potentially saturate quantum information storage capacity.

The paper is organized as follows. In Sec. II, we first present a physical picture of quantum fractal liquids by reviewing how condensation of extended objects emerges in topological spin liquids. This section serves as a nontechnical summary of the paper. We then present a general framework to construct a family of classical fractal liquids in Sec. III. In Sec. IV, we demonstrate that ground states of classical fractal liquids correspond to limit cycles under real-space RG transformations. In Sec. V, we present a general framework 
to construct a family of three-dimensional quantum fractal liquids. In Sec. VI, we discuss quasiparticle properties and look at several examples. In Sec. VII, we briefly discuss coding properties of quantum fractal liquids.

Some comments on the paper follow. We adopt the stability against local perturbations as the definition of topological order. By TQFT, we mean an axiomatic formulation by Atiyah which admits only a finite number of degenerate ground states. ${ }^{14,21}$ By topological spin liquids, we mean gapped spin systems without local symmetries, i.e., topologically ordered spin systems. Discussion on gapless quantum spin liquids is beyond the scope of this paper. Our construction of quantum fractal liquids is theoretically motivated, and its relevance to experimental realization may not be immediately clear. Some technical tools are borrowed from a recent work by Haah. $^{22}$ Glassy dynamics arising in classical and quantum fractal liquids, which is beyond the scope of this paper, may be analyzed via mapping from "classical dynamics" to "static quantum Hamiltonian" as illustrated in Ref. 23.

\section{TOPOLOGICAL SPIN LIQUID}

In conventional topological spin liquids, extended objects with continuous geometries emerge from underlying gauge symmetries. ${ }^{6,7}$ In contrast, quantum fractal liquids are condensation of fractal objects with discrete geometries which emerge from certain algebraic symmetries. Geometric properties of extended objects can be characterized by topological classes of symmetry operators; fractal operators are associated with quantum fractal liquids. In this section, we present a physical picture of quantum fractal liquids.

\section{A. Topological spin liquid and string nets}

We begin with the simplest string-net model, known as $\mathbb{Z}_{2}$ spin liquid (or the toric code) [Fig. 1(a)]. ${ }^{2,24}$ Consider a square lattice where qubits live on edges of the lattice with periodic boundary conditions. The Hamiltonian is

$$
H=-\sum_{s} A_{s}-\sum_{p} B_{p}, \quad A_{s}=\prod_{r \in s} X_{r}, \quad B_{p}=\prod_{r \in p} Z_{r},
$$

where $s$ represents a star and $p$ represents a plaquette. Pauli$X$ and $-Z$ operators act on each qubit as follows: $Z|0\rangle=$ $|0\rangle, Z|1\rangle=-|1\rangle, X|0\rangle=|1\rangle$, and $X|1\rangle=|0\rangle$. The model is exactly solvable as interaction terms $A_{s}$ and $B_{p}$ commute with each other, and ground states satisfy

$$
A_{s}|\psi\rangle=|\psi\rangle, \quad B_{p}|\psi\rangle=|\psi\rangle, \quad \forall s, p .
$$

A ground state can be viewed as condensation of stringlike extended objects. Consider a trivial product state $|0\rangle^{\otimes N}$ over the entire lattice ( $N$ is the total number of qubits) and observe that $B_{p}|0\rangle^{\otimes N}=|0\rangle^{\otimes N}$. The following is a ground state:

$$
\left|\psi_{\text {loop }}\right\rangle=\prod_{s}\left(1+A_{s}\right)|0\rangle^{\otimes N}
$$

since $A_{s}\left(1+A_{s}\right)=1+A_{s}$. The normalization factor is omitted. It is a superposition of $A_{s_{1}} A_{s_{2}} A_{s_{3}} \ldots|0\rangle^{\otimes N}$. Since $A_{s}$ is a product of Pauli- $X$ operators, it flips qubits: $|0\rangle \leftrightarrow|1\rangle$. Then, a term $A_{s}|0\rangle^{\otimes N}$ can be viewed as a state with one small loop on a dual lattice, and a term $A_{s_{1}} A_{s_{2}}|0\rangle^{\otimes N}$ with neighboring stars $s_{1}$ and $s_{2}$ is a state with a larger loop [Fig. 1(b)]. In general, $A_{s_{1}} A_{s_{2}} A_{s_{3}} \ldots|0\rangle^{\otimes N}$ is a state with loops of various sizes and shapes. A ground state is a superposition of all the loop states [Fig. 1(c)]:

$$
\left|\psi_{\text {loop }}\right\rangle=\sum_{\forall \gamma}|\gamma\rangle
$$

where $\gamma$ represents an arbitrary contractible loop configuration. Therefore, a ground state is condensation of fluctuating stringlike objects with $\mathbb{Z}_{2}$ gauge symmetry.

One can construct a general quantum many-body system with several types of strings constrained by discrete gauge symmetry. Levin and Wen derived the most general form of wave functions that are represented as condensation of (a)

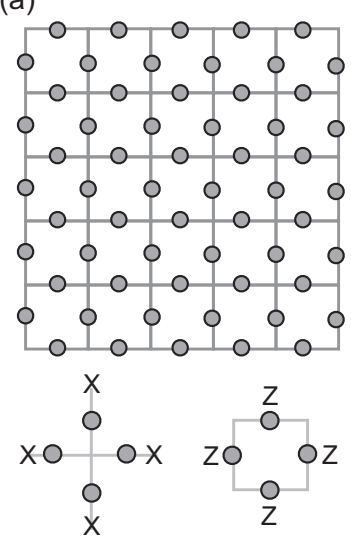

(b)

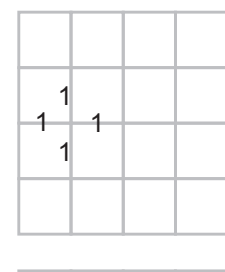

(d) $\ell_{0}^{(Z)}$
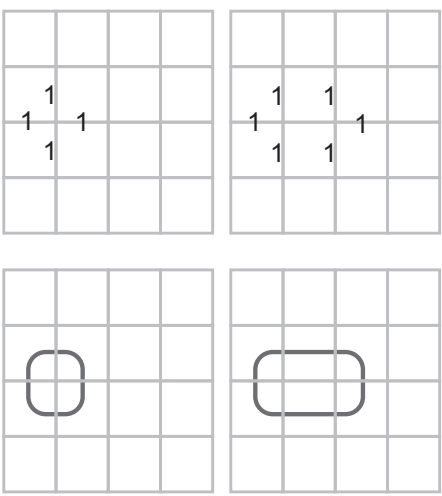
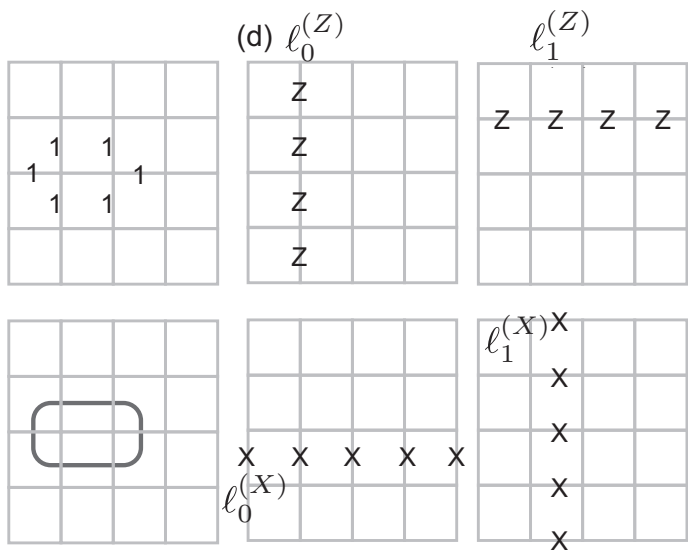

(c)

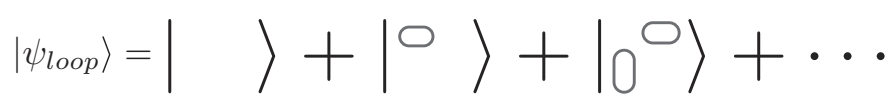

FIG. 1. $\mathbb{Z}_{2}$ spin liquid (the toric code). (a) The Hamiltonian. (b) Loop states on a dual lattice. (c) Condensation of loops. (d) Logical operators. 
stringlike extended objects on a two-dimensional lattice by further assuming that wave functions possess scale invariance and correspond to fixed points of renormalization group (RG) transformations. ${ }^{15}$ Indeed, a ground state of $\mathbb{Z}_{2}$ spin liquid has scale invariance as it is a superposition of loops of all the different sizes and shapes. Note that scale invariance is required for systems described by TQFT since they must be invariant under continuous deformations. Yet, scale invariance is not a necessary condition for the presence of topological order. As we will see, quantum fractal liquids do not have full continuous scale symmetries. Instead, they have discrete scale symmetries where systems are invariant only under a limited set of scale transformations and ground states correspond to limit cycles of RG transformations.

Geometric properties of extended objects can be characterized by topological properties of global symmetry operators. Formally, symmetries of the Hamiltonian can be captured by unitary transformations that leave the Hamiltonian invariant:

$$
U^{\dagger} H U=H \text {. }
$$

Interaction terms $A_{s}$ and $B_{p}$ are symmetry operators for $\mathbb{Z}_{2}$ spin liquid since $\left[A_{s}, H\right]=\left[B_{p}, H\right]=0$ where the groundstate space is an invariant subspace under actions of interaction terms. There also exist nontrivial symmetry operators which act nontrivially inside the ground-state space [see Fig. 1(d)]:

$$
\left[H, \ell_{0}^{(Z)}\right]=\left[H, \ell_{1}^{(Z)}\right]=\left[H, \ell_{0}^{(X)}\right]=\left[H, \ell_{1}^{(X)}\right]=0
$$

with nontrivial winding on a torus. These symmetries are spontaneously broken in ground states.

Since nontrivial symmetry operators commute with the Hamiltonian, they do not change the energy. Yet, they can not be written as products of $A_{s}$ or $B_{p}$ and transform degenerate ground states into each other. Recall that $\left|\psi_{\text {loop }}\right\rangle$ is a condensation of loops that can be shrunk into a vacuum under $\mathbb{Z}_{2}$ gauge symmetry. An application of $\ell_{0}^{(X)}$ to $\left|\psi_{\text {loop }}\right\rangle$ creates a nontrivial loop winding in the $\hat{x}$ direction. Similarly, $\ell_{1}^{(X)}\left|\psi_{\text {loop }}\right\rangle$ is a condensation of loops with nontrivial winding in the $\hat{y}$ direction. Four degenerate ground states may be indexed by winding numbers as $\left|\tilde{\gamma}_{x}\right\rangle \otimes\left|\tilde{\gamma}_{y}\right\rangle$ with $\gamma_{x}, \gamma_{y}=0,1$ where $\gamma_{x}$ and $\gamma_{y}$ represent the absence or presence of windings in the $\hat{x}$ and $\hat{y}$ directions, respectively. Then, nontrivial symmetry operators $\ell_{0}^{(X)}$ and $\ell_{1}^{(X)}$ act like Pauli- $X$ operators on a pair of logical qubits $\left|\tilde{\gamma}_{x}\right\rangle \otimes\left|\tilde{\gamma}_{y}\right\rangle$. It is convenient to represent their commutation relation as follows:

$$
\left\{\begin{array}{ll}
\ell_{0}^{(Z)}, & \ell_{1}^{(Z)} \\
\ell_{0}^{(X)}, & \ell_{1}^{(X)}
\end{array}\right\}
$$

where operators in the same column anticommute with each other while logical operators in different columns commute with each other. (This notation for commutation relations among logical operators will be used throughout the paper.) Anticommuting pairs of nontrivial symmetry operators can be viewed as logical Pauli $Z$ and Pauli $X$ acting on logical qubits $\left|\tilde{\gamma}_{x}\right\rangle \otimes\left|\tilde{\gamma}_{y}\right\rangle$.

These nontrivial symmetry operators are often called logical operators in quantum information community as they are utilized to rewrite encoded logical qubits in the ground-state space. Note the $\mathbb{Z}_{2}$ spin liquid is a good quantum error-correcting code as only global operators with nontrivial winding can change encoded logical qubits. This is an insight on stability against local perturbations in topological phases from a quantum information perspective. . $^{2,25}$

One can generalize the $\mathbb{Z}_{2}$ spin liquid to higher-dimensional systems. For instance, the $D$-dimensional toric code has anticommuting pairs of $m$-dimensional and $(D-m)$-dimensional logical operators:

$$
m \text {-dim } \leftrightarrow(D-m) \text {-dim, } m \text { : integer. }
$$

Its ground states can be viewed as condensation of $m$ dimensional extended objects, or $(D-m)$-dimensional extended objects in a dual description. In general, for a quantum many-body system described by TQFT, extended objects (Wilson loops or Wilson surfaces) have continuous geometries with integer dimensionality. In fact, the dimensional duality of nontrivial symmetry operators is a consequence of the Poincaré duality for systems described by TQFT and can be derived from continuous deformability of logical operators. ${ }^{26}$

\section{B. Emergence of fractal geometry}

In this section, we give a physical picture of quantum fractal liquids by analyzing how fractal geometries arise in classical spin systems. We shall concentrate on a toy model proposed by Newman and Moore ${ }^{19}$ although our arguments are applicable to a broader class of classical fractal liquids which will be introduced in Sec. III. Consider a square lattice where $L \times L$ spins live on vertices. The Hamiltonian is

$$
H=-\sum_{i=0}^{L-2} \sum_{j=0}^{L-1} \Pi_{i j}, \quad \Pi_{i j}=Z_{i, j} Z_{i+1, j} Z_{i+1, j+1}
$$

where $Z_{i j}$ acts on a spin at $(i, j)$ and we represented interaction terms graphically as a matrix. We denote spin values at $(i, j)$ for $i, j=0, \ldots, L-1$ as $s_{i, j}=0,1$. Ground states must satisfy $\Pi_{i j} \psi=\psi$ :

$$
s_{i, j}+s_{i+1, j}=s_{i+1, j+1}(\bmod 2)
$$

for all $i$ and for $j=0, \ldots, L-2$. The following is a ground state:

$$
\left[\begin{array}{ccccccccc}
1 & 0 & 0 & 0 & 0 & 0 & 0 & 0 & \ldots \\
1 & 1 & 0 & 0 & 0 & 0 & 0 & 0 & \ldots \\
1 & 0 & 1 & 0 & 0 & 0 & 0 & 0 & \ldots \\
1 & 1 & 1 & 1 & 0 & 0 & 0 & 0 & \ldots \\
1 & 0 & 0 & 0 & 1 & 0 & 0 & 0 & \ldots \\
1 & 1 & 0 & 0 & 1 & 1 & 0 & 0 & \ldots \\
1 & 0 & 1 & 0 & 1 & 0 & 1 & 0 & \ldots \\
1 & 1 & 1 & 1 & 1 & 1 & 1 & 1 & \ldots \\
\vdots & \vdots & \vdots & \vdots & \vdots & \vdots & \vdots & \vdots & \ddots
\end{array}\right],
$$

where the upper-left corner corresponds to $s_{0,0}$ and the lowerright corner corresponds to $s_{L-1, L-1}$. Configuration of sites with spin value 1 forms the Sierpinski triangle [Fig. 4(a)]. It is interesting to observe that translation symmetries are spontaneously broken in a ground state.

The model has a large number of degenerate ground states. Let us pick up arbitrary spin values on the first row $\vec{s}=\left(s_{0,0}, s_{1,0}, s_{2,0}, \ldots, s_{L-1,0}\right)$. Then, spin values on other rows are determined by Eq. (4). Since there are $2^{L}$ possible choices 
for $\vec{s}$, there are $2^{L}$ degenerate ground states. It is convenient to view the model as a time evolution of one-dimensional cellular automaton where spin values on lower rows are computed via an update rule in Eq. (4). It is well known that one-dimensional cellular automata with linear update rules generate a variety of fractal geometries (see Ref. 27 for a review). One may consider a general class of classical spin models with fractal ground states by designing interaction terms which imitate update rules of one-dimensional cellular automata. See Sec. III for such generalization.

The Sierpinski triangle model has liquidlike order, but is different from conventional classical spin liquids such as antiferromagnetic Ising models on geometrically frustrated lattices. ${ }^{28-32}$ Due to unconventional three-body interactions, the model does not have magnetic order at any temperature including $T=0$. A zero-temperature thermodynamic entropy is large, but not extensive: $S=\sqrt{N}$. Whether the model may select an ordered ground state via an order-by-disorder mechanism is not known. In this paper, we refer to a family of the Sierpinski triangle model as classical fractal liquids despite technical subtleties mentioned above.

The model does not have power-law decay of two-point correlation functions as observed in conventional classical spin liquids. Instead, it has an oscillatory power-law behavior with imaginary scaling dimensions, exhibiting discrete scale symmetries. Consider the following three-point correlation functions:

$$
C(r)=\left\langle Z_{i, j} Z_{i+r, j} Z_{i+r, j+r}\right\rangle .
$$

In the ground space manifold, $C(r)$ is

$$
\begin{aligned}
C(r) & =1, & r=2^{m} \\
& =0, & r \neq 2^{m}
\end{aligned}
$$

with oscillatory behaviors in $\ln r$, instead of $r$. Note we took the average over all the degenerate ground states.

Correlation functions can be computed as follows. ${ }^{33}$ For $r=1, C(r)$ is an expectation value of an interaction term $\Pi_{i, j}=Z_{i, j} Z_{i+1, j} Z_{i+1, j+1}$, so $C(1)=1$. For $r=2$, one has the following identity:

$$
\Pi_{i . j}^{\prime} \equiv Z_{i, j} Z_{i+2, j} Z_{i+2, j+2}=\Pi_{i, j} \Pi_{i+1, j} \Pi_{i+1, j+1} .
$$

So, one has $C(2)=1$. Similarly, three-point correlation functions for $r=4$ can be constructed from those for $r=2$ :

$$
\Pi_{i, j}^{\prime \prime} \equiv Z_{i, j} Z_{i+4, j} Z_{i+4, j+4}=\Pi_{i, j}^{\prime} \Pi_{i+2, j}^{\prime} \Pi_{i+2, j+2}^{\prime}
$$

and $C(4)=1$. Iterating these arguments, one finds $C(r)=1$ for $r=2^{m}$. For $r \neq 2^{m}$, three-point correlation terms can not be written as a product of interaction terms, so $C(r)=0$.

At finite temperature, the three-point correlation function reads as

$$
C(r)=(1-2 p)^{r^{\ln 3 / \ln 2}}, \quad r=2^{m}
$$

with $p=e^{-\beta} /\left(e^{-\beta}+e^{\beta}\right)$ where the exponent depends on the fractal dimension. This is because $C\left(2^{m}\right)$ is an expectation value of a product of $3^{m}\left(=r^{\ln 3 / \ln 2}\right)$ interaction terms. The correlation function for all $r$ may be written as

$$
C(r) \propto \exp \left(- \text { const } \times r^{\ln 3 / \ln 2}\right) \sum_{k=-\infty}^{\infty} r^{i \frac{2 \pi k}{\ln 2}},
$$

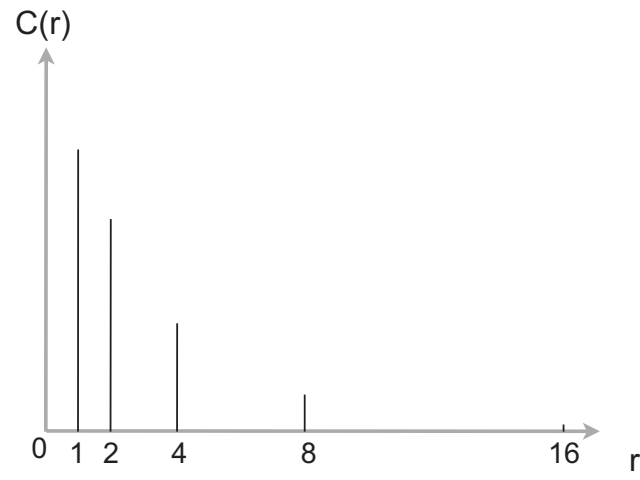

FIG. 2. Discrete scale symmetries and imaginary scaling dimensions in three-point correlation function.

where oscillatory behaviors in $\ln r$ are represented by power law. Note the imaginary scaling dimension (see Fig. 2) is characteristic of systems with discrete scale symmetries as pointed out by Wilson. ${ }^{34}$

Geometric properties of degenerate ground states in the Sierpinski triangle model can be captured by geometric shapes of logical operators:

$\ell=\left[\begin{array}{ccccc}Z & I & I & I & \ldots \\ I & I & I & I & \ldots \\ I & I & I & I & \ldots \\ I & I & I & I & \ldots \\ \vdots & \vdots & \vdots & \vdots & \ddots\end{array}\right], \quad r=\left[\begin{array}{ccccc}X & I & I & I & \ldots \\ X & X & I & I & \ldots \\ X & I & X & I & \ldots \\ X & X & X & X & \ldots \\ \vdots & \vdots & \vdots & \vdots & \ddots\end{array}\right]$.

So, it has the following pairs of logical operators:

$$
0 \text {-dim } \leftrightarrow \frac{\ln 3}{\ln 2} \text {-dim. }
$$

While the model has a fractal logical operator, a ground state does not have any quantum fluctuation, and is not topologically ordered since its partner is a trivial logical operator with zerodimensional geometry. To have topological order, both logical operators must have topologically nontrivial geometries (i.e., they must be finite dimensional). In the remainder of the paper, we present a large class of quantum spin systems which have pairs of anticommuting fractal logical operators:

$$
\text { fractal-dim } \leftrightarrow \text { fractal-dim. }
$$

\section{Topological phase transition}

A quantum many-body system with topological order can be viewed as condensation of extended objects with a variety of geometric shapes. It is natural to expect that two ground states with different types of extended objects belong to different topological phases. In this section, we make this intuition more precise by arguing that two spin systems with topologically different classes of logical operators are separated by quantum phase transitions.

Quantum phases are characterized by long-range entanglement of a many-body quantum system with mass gap at zero temperature. Ground states in different quantum phases can not be connected continuously at the thermodynamic limit. Let us consider two ground states $\left|\psi_{A}\right\rangle$ and $\left|\psi_{B}\right\rangle$ of two 
(a)

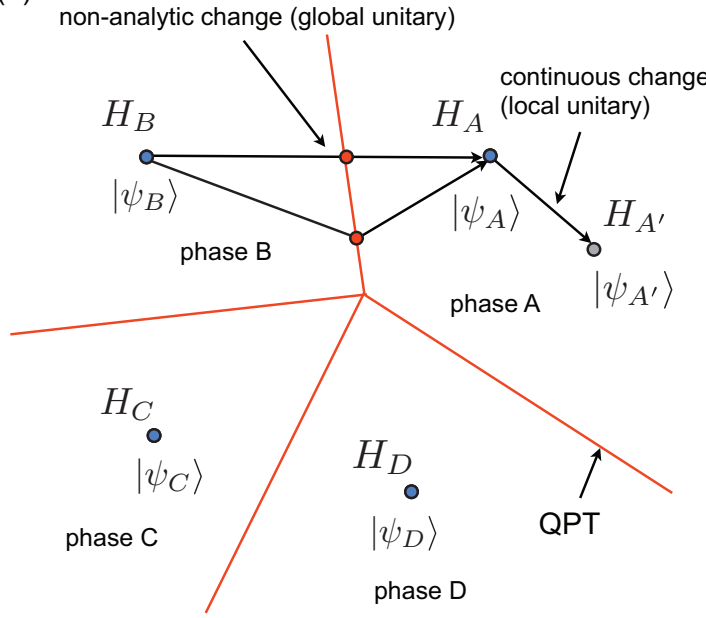

(b)

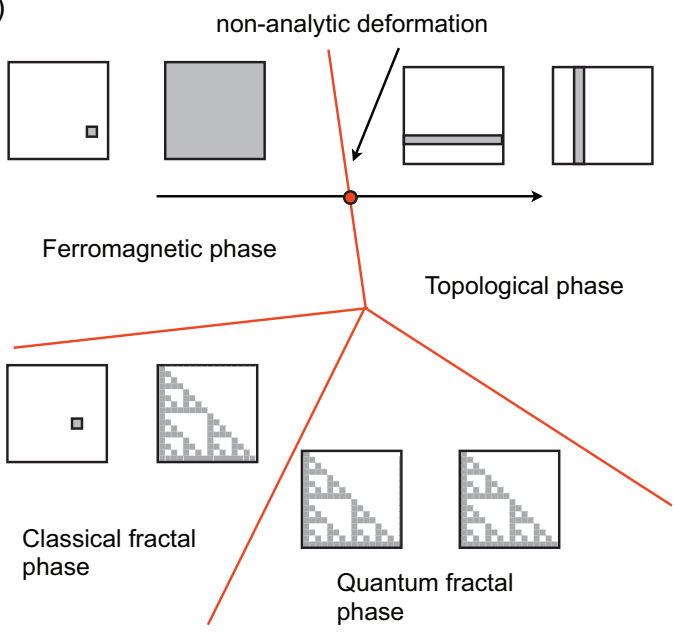

FIG. 3. (Color online) (a) Continuous deformability of ground states. (b) Continuous deformability of logical operators.

different gapped Hamiltonians $H_{A}$ and $H_{B}$ and ask if they are separated by quantum phase transitions (nonanalytic changes of ground-state properties). Two ground states $\left|\psi_{A}\right\rangle$ and $\left|\psi_{B}\right\rangle$ are said to be in different quantum phases when there always exist quantum phase transitions between $H_{A}$ and $H_{B}$ regardless of paths between $H_{A}$ and $H_{B}$. Conversely, if there exists a continuous change from $H_{A}$ to $H_{B}$ without crossing quantum phase transitions, two ground states $\left|\psi_{A}\right\rangle$ and $\left|\psi_{B}\right\rangle$ are in the same quantum phase.

An equivalent, but more convenient, way of classifying quantum phases uses local unitary transformations. Two ground states $\left|\psi_{A}\right\rangle$ and $\left|\psi_{B}\right\rangle$ are considered to be in the same quantum phase when there exists some local unitary transformation connecting $\left|\psi_{A}\right\rangle$ and $\left|\psi_{B}\right\rangle$. By local unitary transformations, we mean transformations generated by a set of geometrically local quantum operations, applied for a finite duration. On the other hand, when there is no local unitary transformation connecting $\left|\psi_{A}\right\rangle$ and $\left|\psi_{B}\right\rangle$, they are in different quantum phases. Only global unitary transformations can change long-range entanglement of ground states.

These two classification principles of quantum phases are equivalent under appropriate assumptions. If two gapped Hamiltonians $H_{A}$ and $H_{B}$ can be transformed into each other continuously without closing the energy gap, correlation lengths of ground states remain finite, and ground states at each stage of transformation can be approximated via some quasilocal unitary transformations applied to original ground states. ${ }^{25}$ Conversely, if $\left|\psi_{A}\right\rangle$ and $\left|\psi_{B}\right\rangle$ are connected by some local unitary transformations, one can always continuously transform $H_{A}$ into $H_{B}$.

The classification of quantum phases, based on continuous deformability of ground-state wave functions, reminds us of the study of topology in mathematics, which aims to classify geometric shapes of objects based on continuous deformability. Roughly speaking, two objects are considered to be equivalent when they can be transformed into each other via continuous deformations (diffeomorphism). Yet, if one can not continuously deform an object to the other, they are considered to be topologically different. The similarity between classifications of quantum phases, based on continuous deformability of wave functions, and classifications of geometric shapes, based on continuous deformability of geometric objects, allows us to use the notion of topology in classifying quantum phases (see Fig. 3). Indeed, the following relation holds:

Logical operators are topologically different. $\Rightarrow$ Two systems belong to different quantum phases.

The argument roughly goes as follows. ${ }^{35,36}$ Consider two systems with topologically distinct logical operators $\ell$ and $\ell^{\prime}$. Let us suppose that they belong to the same quantum phase. Then, there must be some local unitary transformation $U$ such that $U \ell U^{\dagger}=\ell^{\prime}$. Yet, this is not possible since local unitary transformation can change geometric shapes of logical operators only continuously at the thermodynamic limit. Therefore, models with topologically different types of logical operators belong to different quantum phases and are always separated by quantum phase transitions. Note that there is no local unitary that transforms a stringlike logical operator to a fractal logical operator and, thus, fractal models are different from conventional topologically ordered systems. " $\Leftarrow$ " of the above relation is proven only for stabilizer Hamiltonians in two dimensions. ${ }^{36}$

A naturally arising question is whether quantum fractal liquids may be viewed as stable quantum phases or not. Indeed, the model Hamiltonians of quantum fractal liquids, proposed in this paper, involve fine-tuned interaction terms, and whether quantum fractal order physically emerges with generic interaction terms may not be immediately clear. In order for these toy models of exotic topological order to serve as representatives of realistic quantum phases, they must possess stability against deformations of Hamiltonians. It turns out that quantum fractal phases are stable against any types of sufficiently small but finite perturbations. To be more precise, consider a model Hamiltonian $H_{0}$ and arbitrary local perturbation $V$ :

$$
H(\epsilon)=H_{0}+\epsilon V \text {. }
$$

Here, we do not assume any symmetry constraints on $V$. (For instance, $V$ can be time-reversal-symmetry breaking 
(a)

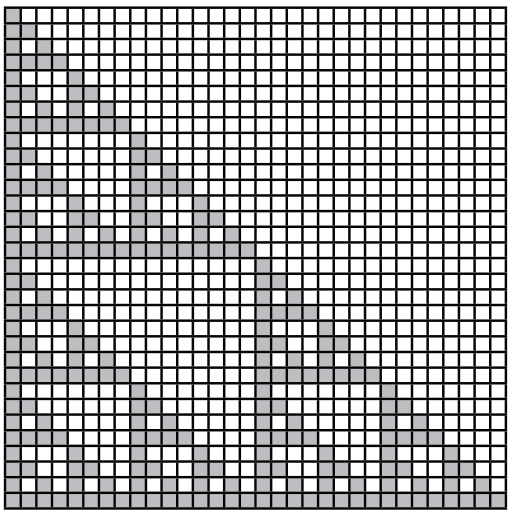

(b)

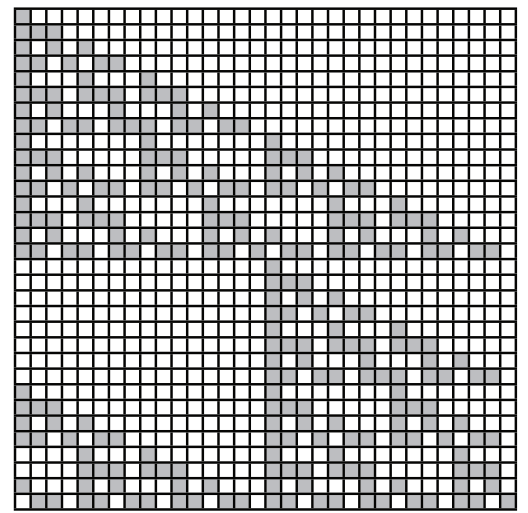

(c)

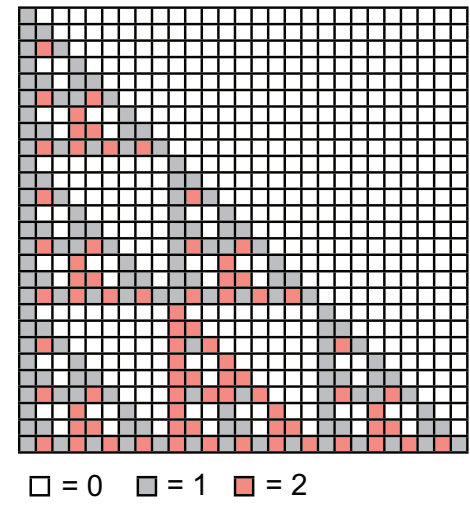

FIG. 4. (Color online) (a) The Sierpinski triangle from $f=1+x$ over $\mathbb{F}_{2}$. (b) The Fibonacci model from $f=1+x+x^{2}$ over $\mathbb{F}_{2}$. (c) The generalized Sierpinski triangle from $f=1+x$ over $\mathbb{F}_{3}$.

and nonuniform.) By local perturbation, we mean that $V$ consists only of terms that act on spins inside finite regions with bounded norms. Examples of such perturbation include uniform or disordered magnetic fields and two-body Ising or Heisenberg interactions. Then, we claim that there exists $\epsilon_{c}>0$ such that the ground-state properties of the model Hamiltonian $H_{0}$ is stable for $0 \leqslant \epsilon<\epsilon_{c}$. Stability of quantum fractal liquids originates from the fact that they work as quantum error-correcting code which securely store quantum information in the presence of noises. See Sec. V for detailed arguments.

The stability of quantum fractal phases can be physically argued as follows. Let $\left|\psi_{j}\right\rangle$ be degenerate ground states of unperturbed Hamiltonian $H_{0}$. In order to transform one ground state to other ground states, one needs to apply fractal logical operators which have supports on a large number of spins that scale up with the system size. Since a perturbation $V$ consists only of local terms, only extremely higher-order perturbative contributions may couple degenerate ground states, and effective coupling strengths are exponentially suppressed. So, the energy splitting between degenerate ground states is exponentially suppressed, and the ground-state degeneracy is protected at the thermodynamic limit for sufficiently small but finite $\epsilon$. Similarly, one can argue that the energy gap between excited states and the ground states remains finite. Provided that the energy gap and the degeneracy are protected, one can find a quasilocal unitary transformation $U$ that maps unperturbed ground states to perturbed ground states:

$$
\left|\psi_{j}(\epsilon)\right\rangle=U\left|\psi_{j}\right\rangle
$$

provided $\epsilon$ is sufficiently small. Therefore, $H(\epsilon)$ and $H_{0}$ belong to the same quantum phase, and $H_{0}$ serves as a representative of quantum phases, being at limit cycles of RG transformations. See Ref. 25 for rigorous proof.

In summary, we expect that there will be four classes of quantum phases arising in gapped spin systems:

(a) Ferromagnetic phase: 0 -dim $\leftrightarrow D$-dim.

(b) Classical fractal phase: 0 -dim $\leftrightarrow$ fractal-dim.

(c) Topological phase: $m$-dim $\leftrightarrow D-m$-dim $(m>0)$.

(d) Quantum fractal phase: fractal-dim $\leftrightarrow$ fractal-dim.

\section{CLASSICAL FRACTAL LIQUID}

\section{A. Fractal and algebraic symmetry}

In this section, we construct a family of classical fractal liquids. We begin with polynomial representation of the Sierpinski triangle [Fig. 4(a)]. Consider a polynomial $f=$ $1+x$ over $\mathbb{F}_{2}$ and its powers:

$$
\begin{aligned}
& f^{0}=1, \quad f^{1}=1+x, \quad f^{2}=1+x^{2}, \\
& f^{3}=1+x+x^{2}+x^{3}, \quad f^{4}=1+x^{4}, \\
& f^{5}=1+x+x^{4}+x^{5},
\end{aligned}
$$

where coefficients are computed modulo 2. More graphically, one has

$$
\left[\begin{array}{l}
f^{0} \\
f^{1} \\
f^{2} \\
f^{3} \\
f^{4} \\
f^{5}
\end{array}\right]=\left[\begin{array}{llllll}
1 & & & & & \\
1 & x & & & & \\
1 & & x^{2} & & & \\
1 & x & x^{2} & x^{3} & & \\
1 & & & & x^{4} & \\
1 & x & & & x^{4} & x^{5}
\end{array}\right]
$$

where the Sierpinski triangle emerges in a geometric pattern of nonzero coefficients in $f^{j}$.

The entire Sierpinski triangle can be represented as a single polynomial with $x$ and $y$ :

$$
\mathbf{f}(x, y)=1+f y+f^{2} y^{2}+f^{3} y^{3}+\cdots,
$$

where the $j$ th row is indexed by $y^{j}$. More graphically, one has

$$
\mathbf{f}(x, y)=\left[\begin{array}{llllll}
1 & & & & & \\
y & x y & & & & \\
y^{2} & & x^{2} y^{2} & & & \\
y^{3} & x y^{3} & x^{2} y^{3} & x^{3} y^{3} & & \\
y^{4} & & & & x^{4} y^{4} & \\
y^{5} & x y^{5} & & & x^{4} y^{5} & x^{5} y^{5}
\end{array}\right],
$$

where nonzero coefficients of $x^{i} y^{j}$ correspond to filled elements of the Sierpinski triangle at $(i, j)$. 
Another interesting example of fractal geometries is generated by $f=1+x+x^{2}$ over $\mathbb{F}_{2}$ :

$$
\left[\begin{array}{l}
f^{0} \\
f^{1} \\
f^{2} \\
f^{3} \\
f^{4}
\end{array}\right]=\left[\begin{array}{llllllll}
1 & & & & & & & \\
1 & x & x^{2} & & & & & \\
1 & & x^{2} & & x^{4} & & & \\
1 & x & & x^{3} & & x^{5} & x^{6} & \\
1 & & & & x^{4} & & & x^{8}
\end{array}\right] .
$$

Again, the entire fractal geometry can be represented as $\mathbf{f}(x, y)=1+f y+f^{2} y^{2}+f^{3} y^{3}+\cdots$. The model is often called the Fibonacci model since its fractal dimension is given by $\frac{\ln 1+\sqrt{5}}{\ln 2}$ [Fig. 4(b)]. These constructions can be generalized to polynomials over $\mathbb{F}_{p}(p>2)$ with an arbitrary prime $p$. For instance, $f=1+x$ over $\mathbb{F}_{3}(p=3)$ leads to a generalization of the Sierpinski triangle for three-dimensional spins [Fig. 4(c)]:

$$
\left[\begin{array}{l}
f^{0} \\
f^{1} \\
f^{2} \\
f^{3} \\
f^{4} \\
f^{5}
\end{array}\right]=\left[\begin{array}{cccccc}
1 & & & & & \\
1 & x & & & & \\
1 & 2 x & x^{2} & & & \\
1 & & & x^{3} & & \\
1 & x & & x^{3} & x^{4} & \\
1 & 2 x & x^{2} & x^{3} & 2 x^{4} & x^{5}
\end{array}\right] .
$$

The self-similarity in fractal geometries arises from discrete scale symmetries of generating polynomials. Consider an arbitrary polynomial $f$ over $\mathbb{F}_{p}$ with prime $p$ :

$$
f=c_{0}+c_{1} x+c_{2} x^{2}+c_{3} x^{3}+\cdots,
$$

where $c_{j}=0, \ldots, p-1$. Then, its $p$ th power is

$$
f^{p}=c_{0}+c_{1} x^{p}+c_{2} x^{2 p}+c_{3} x^{3 p}+\cdots .
$$

For instance, with $f=1+2 x+x^{2}$ over $\mathbb{F}_{3}$, one finds

$$
\begin{aligned}
f & =1+2 x+x^{2}, \quad f^{3}=1+2 x^{3}+x^{6}, \\
f^{9} & =1+2 x^{9}+x^{18} .
\end{aligned}
$$

So, generated fractal geometry $\mathbf{f}(x, y)=1+f y+f^{2} y^{2}+$ $f^{3} y^{3}+\cdots$ has a self-similarity where the same pattern appears repeatedly at different length scales.

Fractal geometries do not possess gauge symmetries since growth of filled elements violates charge conservation where a single element may evolve into multiple elements of the same type in the $\hat{y}$ direction. This is in strong contrast with the fact that continuous geometries often have physical interpretations based on conservation laws associated with underlying gauge symmetries as in the case of TQFT. ${ }^{15}$ Charge conservation in scale-invariant spin models originates from group theoretical constraints imposed on the parent Hamiltonian. Fractal geometries obey a more general form of symmetries, which are referred to as algebraic symmetries in this paper, due to a possible relation to the theory of algebraic geometry which concerns geometric structures of solutions of polynomial equations.

\section{B. Polynomial representation of Pauli operators}

To construct parent Hamiltonians of classical fractal liquids, it is convenient to represent interaction terms by polynomials too. Note this is a standard technique in classical coding theory. ${ }^{37}$ Consider a polynomial $f$ over $\mathbb{F}_{2}$ :

$$
f=\sum_{j=-\infty}^{\infty} c_{j} x^{j}, \quad c_{j}=0,1 .
$$

We define the corresponding Pauli operators as follows:

$$
Z(f)=\prod_{j=-\infty}^{\infty} Z_{j}^{c_{j}}, \quad X(f)=\prod_{j=-\infty}^{\infty} X_{j}^{c_{j}},
$$

where $Z_{j}$ and $X_{j}$ are Pauli operators acting on the $j$ th qubit. So, a polynomial $f$ encodes positions of qubits where Pauli operators $Z_{j}$ or $X_{j}$ may act. For instance, $f=1+x+x^{2}$ and $Z(f)=Z_{0} Z_{1} Z_{2}$.

The polynomial representation of Pauli operators is particularly useful for studying spin systems with translation symmetries since translations can be concisely represented in terms of polynomials. For instance, consider a Pauli operator $Z(f)=Z_{0} Z_{1} Z_{2}$ for $f=1+x+x^{2}$. Then, its translation in the $\hat{x}_{+}$direction is given by $Z_{1} Z_{2} Z_{3}$, whose polynomial representation is $Z(x f)$ :

$$
\begin{aligned}
f & =1+x+x^{2} \rightarrow x f=x+x^{2}+x^{3}, \\
Z(f) & =Z_{0} Z_{1} Z_{2} \rightarrow Z(x f)=Z_{1} Z_{2} Z_{3} .
\end{aligned}
$$

In general, $Z(x f)$ is a translation of $Z(f)$ in the $\hat{x}_{+}$direction. Similarly, a translation in the $\hat{x}_{-}$direction is given by $Z\left(x^{-1} f\right)$. One may generalize this formalism to higher-dimensional systems by adding extra variables $y, z, \ldots$.

To gain more insights, let us represent the one-dimensional ferromagnet by polynomials over $\mathrm{F}_{2}$ :

$$
H=-\sum_{j} Z\left[x^{j}(1+x)\right]
$$

where $Z\left[x^{j}(1+x)\right]=Z_{j} Z_{j+1}$. The Sierpinski triangle model, introduced in the previous section, is

$$
H=-\sum_{i j} Z\left[x^{i} y^{j}(1+x+x y)\right],
$$

where interaction terms are translations of $Z(1+x+x y)$. In general, one may consider a classical translation symmetric Hamiltonian

$$
H=-\sum_{i, j, \ldots} Z\left(x^{i} y^{j} \ldots \alpha\right)
$$

with an arbitrary polynomial $\alpha(x, y, \ldots)$. Ground states obey

$$
Z\left(x^{i} y^{j} \ldots \alpha\right)|\psi\rangle=|\psi\rangle, \quad \forall i, j, \ldots
$$

The polynomial representation of Pauli operators becomes particularly powerful in analyzing commutation relations between Pauli operators $Z(f)$ and $X(g)$. Since we are interested in translation-symmetric systems, we want to obtain commutation relations between $Z(f)$ and translations of $X(g)$. Let us imagine that we check commutation relations between $Z(f)$ and $X\left(x^{j} g\right)$ for all $j$ and assign integers $d_{j}=0,1$ as follows:

$$
\begin{array}{lll}
d_{j}=0 & \text { for } & {\left[Z(f), X\left(x^{j} g\right)\right]=0,} \\
d_{j}=1 & \text { for } & \left\{Z(f), X\left(x^{j} g\right)\right\}=0 .
\end{array}
$$


Based on $d_{j}$, we define the commutation polynomial $P(f, g)$ as follows:

$$
P(f, g)=\sum_{j} d_{j} x^{j}
$$

such that

$$
Z(f) X\left(x^{j} g\right)=(-1)^{d_{j}} X\left(x^{j} g\right) Z(f) .
$$

Thus, the commutation polynomial $P(f, g)$ is a collection of commutation relations between $Z(f)$ and $X\left(x^{j} g\right)$. For instance, with $f=1+x+x^{2}$ and $g=1+x, Z(f)$ anticommutes only with $X\left(x^{-1} g\right)$ and $X\left(x^{2} g\right)$. So, the commutation polynomial is $P(f, g)=x^{-1}+x^{2}$.

The commutation polynomial $P(f, g)$ can be concisely written by introducing the notion of dual:

$$
f=\sum_{j=-\infty}^{\infty} c_{j} x^{j} \rightarrow \bar{f}=\sum_{j=-\infty}^{\infty} c_{j} x^{-j},
$$

where the dual $\bar{f}$ is obtained by taking $x \rightarrow x^{-1}$. Then, the commutation polynomial is given by the convolution

$$
P(f, g)=f \bar{g} .
$$

For instance, one has $f \bar{g}=\left(1+x+x^{2}\right)\left(1+x^{-1}\right)=x^{-1}+$ $2+2 x+x^{2}=x^{-1}+x^{2}$ for the above example. The proof of Eq. (16) is straightforward by explicit calculation. Generalization to polynomials over $\mathbb{F}_{p}$ is also straightforward by using generalized Pauli matrices for $\mathbb{Z}_{p}$.

Periodic boundary conditions can be introduced by imposing $x^{L}=1$. Below, reversibility of polynomial $f(x)$ becomes important. Let $f=\sum_{j} c_{j} x^{j}$ over $\mathbb{F}_{p}$. When $L=p^{m}$, one has

$$
f^{L}=\sum_{j} c_{j} x^{L j}=\sum_{j} c_{j}=f(1)
$$

due to discrete scale symmetries and $x^{L}=1$. A polynomial $f$ is reversible if and only if $f(1) \neq 0$. We say that $f$ is properly normalized when $f(1)=1$ so that $f^{L}=1$.

\section{Classical fractal liquid}

We present general construction of classical fractal liquids. Consider a two-dimensional square lattice with $L \times L$ spins $\left(L=2^{m}\right)$ over $\mathbb{F}_{2}$. The Hamiltonian is

$$
H=-\sum_{i j} Z\left(x^{i} y^{j} \bar{\alpha}\right), \quad \alpha=1-f(x) y
$$

where $f(x)$ is an arbitrary polynomial over $\mathbb{F}_{2}$ with $x$ only. We put periodic boundary conditions both in the $\hat{x}$ and $\hat{y}$ directions, and assume that $f(x)$ is reversible and properly normalized.

In finding ground states of the Hamiltonian, it is convenient to find its logical operators. $Z$-type logical operators are trivial single Pauli operators $\ell_{j}^{(Z)}=Z_{j 0}=Z\left(x^{j}\right)$ for $j=0, \ldots, L-$ 1 while $X$-type logical operators have fractal geometries:

$$
\ell_{j}^{(X)}=X\left(x^{j} \mathbf{f}(x, y)\right), \quad \mathbf{f}(x, y)=1+f y+\cdots+(f y)^{L-1} .
$$

One can see that $\ell_{j}^{(X)}$ commute with all the stabilizer generators since the commutation polynomial between $Z(1+\bar{f} \bar{y})$ and $X(\mathbf{f}(x, y))$ is

$$
(1-\bar{f} \bar{y})\left[1+\bar{f} \bar{y}+\cdots+(\bar{f} \bar{y})^{L-1}\right]=0
$$

when $f(x)$ is properly normalized. We list all the logical operators as follows:

$$
\left\{\begin{array}{l}
\ell_{0}^{(X)}, \ldots, \ell_{L-1}^{(X)} \\
\ell_{0}^{(Z)}, \ldots, \ell_{L-1}^{(Z)}
\end{array}\right\}
$$

So, there are $k=L$ logical bits in total.

By using $X$-type logical operators $\ell_{j}^{(X)}$, one can find all the ground states of a classical fractal liquid. Let us denote spin values at $(i, j)$ as $s_{i j}=0,1$ for $i, j=0, \ldots, L-1$, and represent a ground state $\psi$ as

$$
\psi=\sum_{i j} s_{i j} x^{i} y^{j}
$$

Since the Hamiltonian consists only of $Z$-type Pauli operators, $\psi=0$ with $s_{i j}=0$ is a ground state of the Hamiltonian. (Recall $Z|0\rangle=|0\rangle$ and $Z|1\rangle=-|1\rangle$ in our notation.) To find another ground state, one applies $\ell_{0}^{(X)}$ to $\psi=0$ and obtains a fractal ground state

$$
\psi(1)=1+f y+\cdots+(f y)^{L-1}=\mathbf{f}(x, y) .
$$

One can find all the other ground states by applying fractal logical operators $\ell_{j}^{(X)}$. There are $2^{L}$ degenerate ground states, represented by

$$
\psi(\gamma)=\gamma(x) \mathbf{f}(x, y)
$$

where $\gamma(x)$ is an arbitrary polynomial with $x$ only. Noting $\operatorname{dim} \gamma=L$, one finds $k=L$.

Classical fractal liquids discussed so far are based on first-order cellular automata whose present states at $t=$ $\tau$ depend on states at $t=\tau-1$. In higher-order cellular automata, the present states at $t=\tau$ may depend on states at $t=\tau-q, \ldots, \tau-1$ for $q>1$. One can construct classical fractal liquids based on higher-order cellular automata by taking

$$
\alpha=1+f_{1}(x) y+f_{2}(x) y^{2}+\cdots+f_{q}(x) y^{q} .
$$

However, it is generally difficult to write spin configurations of higher-order classical fractal liquids explicitly.

Since the model does not have gauge symmetries, its quasiparticle excitations violate charge conservation and propagate according to algebraic symmetries imposed by generating polynomial $f(x)$. Recall that ground states $\psi$ satisfy $Z\left(x^{i} y^{j} \bar{\alpha}\right) \psi=\psi$ for all $i, j$, and quasiparticle excitations may be viewed as violations of these algebraic constraints. It is convenient to represent positions of excitations by an excitation polynomial

$$
E(x, y)=\sum_{i, j, \ell} c_{i j} x^{i} y^{j},
$$

where an excited state $\psi^{\prime}$ has

$$
\begin{array}{ll}
c_{i j}=0 & \text { for } Z\left(x^{i} y^{j} \bar{\alpha}\right) \psi^{\prime}=+\psi^{\prime}, \\
c_{i j}=1 & \text { for } Z\left(x^{i} y^{j} \bar{\alpha}\right) \psi^{\prime}=-\psi^{\prime}
\end{array}
$$

such that a quasiparticle is present at $(i, j)$ if and only if $c_{i j}=1$.

Excitations in classical spin liquids are caused by Pauli$X$ spin flips. Consider quasiparticle excitations caused by $X(e(x, y))$ where $e(x, y)$ are polynomials representing positions of spin flips. Since anticommutations between $e(x, y)$ 


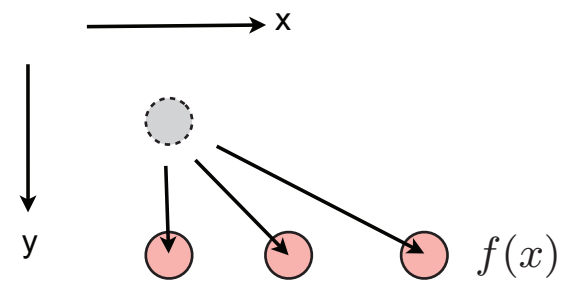

FIG. 5. (Color online) Propagation of quasiparticle excitations.

and $Z\left(x^{i} y^{j} \bar{\alpha}\right)$ create quasiparticles at $(i, j)$, the excitation polynomial is

$$
E(x, y)=e(x, y) \alpha .
$$

For instance, if $X_{0,0}$ with $e=1$ applied, one has multiple excitations $E(x, y)=\alpha$. Consider an isolated excitation at $(0,0)$. An application of $X_{0,0}$ makes it propagate in the $\hat{y}$ direction to multiple excitations represented by $f(x) y$ (Fig. 5). So, quasiparticle excitations propagate via applications of $f(x)$ like time evolution of one-dimensional cellular automaton. Since the model does not have gauge symmetries, one can not associate conserved charge to quasiparticle excitations. Indeed, a single quasiparticle may split into multiple quasiparticles of the same type.

\section{LIMIT CYCLE UNDER RG TRANSFORMATION}

In this section, we demonstrate that ground states of classical fractal liquids correspond to limit cycles under real-space RG transformations. Treatment in this section can be applied to quantum fractal liquids too.

\section{A. Discrete scale symmetry}

Let us consider the Sierpinski triangle model $(f=1+x)$ for $L=8$. A ground state is

$$
\psi=\left[\begin{array}{llllllll}
1 & 0 & 0 & 0 & 0 & 0 & 0 & 0 \\
1 & 1 & 0 & 0 & 0 & 0 & 0 & 0 \\
1 & 0 & 1 & 0 & 0 & 0 & 0 & 0 \\
1 & 1 & 1 & 1 & 0 & 0 & 0 & 0 \\
1 & 0 & 0 & 0 & 1 & 0 & 0 & 0 \\
1 & 1 & 0 & 0 & 1 & 1 & 0 & 0 \\
1 & 0 & 1 & 0 & 1 & 0 & 1 & 0 \\
1 & 1 & 1 & 1 & 1 & 1 & 1 & 1
\end{array}\right]
$$

RG transformations, denoted by $\mathrm{RG}_{i j}(i, j=0,1)$, pick up spins at $(x, y)$ with $x=i(\bmod 2)$ and $y=j(\bmod 2)$, and throw away the rest:

$$
\begin{aligned}
& \mathrm{RG}_{0,0}(\psi)=\left[\begin{array}{cccc}
1 & 0 & 0 & 0 \\
1 & 1 & 0 & 0 \\
1 & 0 & 1 & 0 \\
1 & 1 & 1 & 1
\end{array}\right], \quad \mathrm{RG}_{0,1}(\psi)=\left[\begin{array}{llll}
0 & 0 & 0 & 0 \\
0 & 0 & 0 & 0 \\
0 & 0 & 0 & 0 \\
0 & 0 & 0 & 0
\end{array}\right], \\
& \mathrm{RG}_{1,0}(\psi)=\left[\begin{array}{cccc}
1 & 0 & 0 & 0 \\
1 & 1 & 0 & 0 \\
1 & 0 & 1 & 0 \\
1 & 1 & 1 & 1
\end{array}\right], \quad \mathrm{RG}_{1,1}(\psi)=\left[\begin{array}{llll}
1 & 0 & 0 & 0 \\
1 & 1 & 0 & 0 \\
1 & 0 & 1 & 0 \\
1 & 1 & 1 & 1
\end{array}\right] \text {. }
\end{aligned}
$$

All the RG'ed states are ground states of the Hamiltonian for $L=4$. Let us look at another ground state

$$
\psi=\left[\begin{array}{llllllll}
1 & 1 & 0 & 0 & 0 & 0 & 0 & 0 \\
1 & 0 & 1 & 0 & 0 & 0 & 0 & 0 \\
1 & 1 & 1 & 1 & 0 & 0 & 0 & 0 \\
1 & 0 & 0 & 0 & 1 & 0 & 0 & 0 \\
1 & 1 & 0 & 0 & 1 & 1 & 0 & 0 \\
1 & 0 & 1 & 0 & 1 & 0 & 1 & 0 \\
1 & 1 & 1 & 1 & 1 & 1 & 1 & 1 \\
0 & 0 & 0 & 0 & 0 & 0 & 0 & 0
\end{array}\right]
$$

and its RG transformations

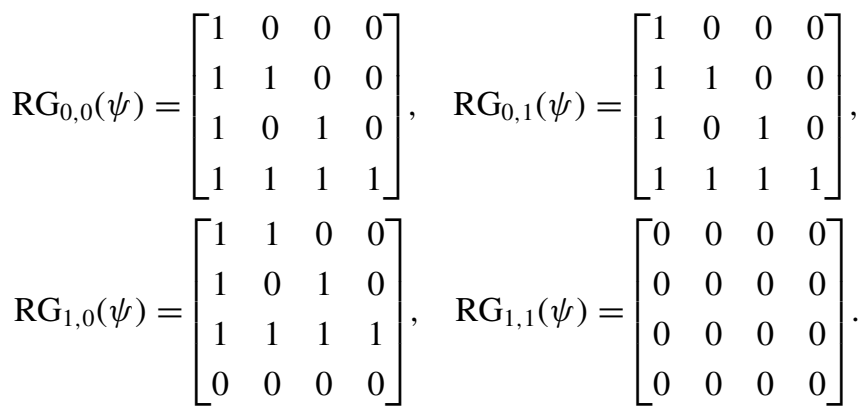

Again, RG'ed states are ground states of a smaller system.

In general, we consider the Sierpinski triangle model for $L=2^{m}$ and $\psi \in \mathcal{G}_{m}$ where $\mathcal{G}_{m}$ is a set of ground states. We view an arbitrary linear map $\Gamma$ from $2^{a} \times 2^{a}$ spins to a single spin as a RG function (Fig. 6):

$$
\Gamma:\left(\mathbb{F}_{2}\right)^{\otimes 2^{2 a}} \rightarrow \mathbb{F}_{2},
$$

where $\Gamma$ maps a wave function for $L=2^{m}$ to a wave function for $L=2^{m-a}$. Then, one has

$$
\Gamma(\psi) \in \mathcal{G}_{m-a} \quad \forall \psi \in \mathcal{G}_{m},
$$

where $\mathcal{G}_{m-a}$ is a set of ground states for $L=2^{m-a}$. One can find $\mathrm{RG}$ transformations that are stable against small perturbations added to wave functions so that it makes sense to discuss how wave functions flow under RG transformations.

Ground states of the Sierpinski triangle model behave nicely under scale transformations by a factor of 2 only. If one performs a similar RG transformation by an incommensurate factor, RG'ed states are not ground states of the Hamiltonian anymore and flow to something else. See Fig. 7 for RG by factors of $\lambda=3^{m}$ where density of 1 states decreases. So, the model has scale symmetries under some limited set of scale transformations. This is a striking contrast with the fact that a ferromagnet, a spin model with continuous scale symmetries, looks always the same under any scale transformations. Note

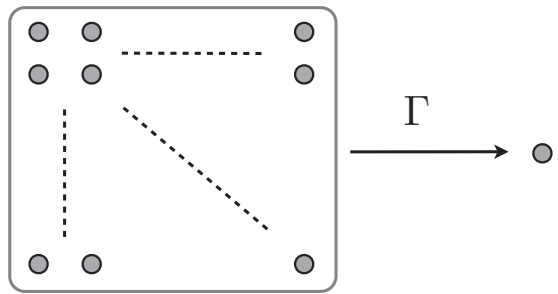

FIG. 6. RG transformation as a linear map $\Gamma$. 
(a)

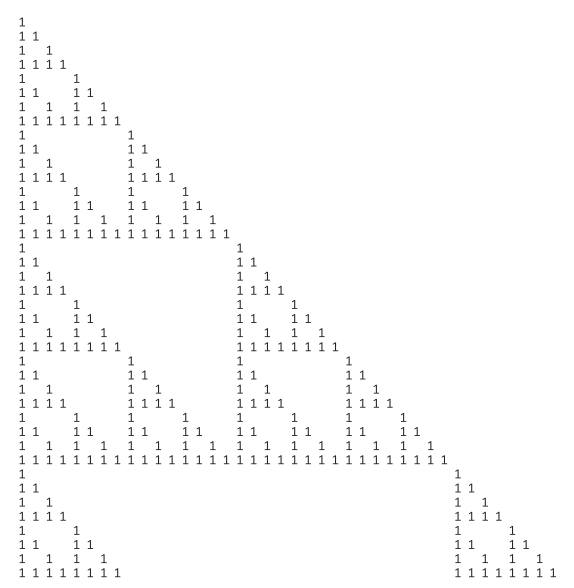

(d)

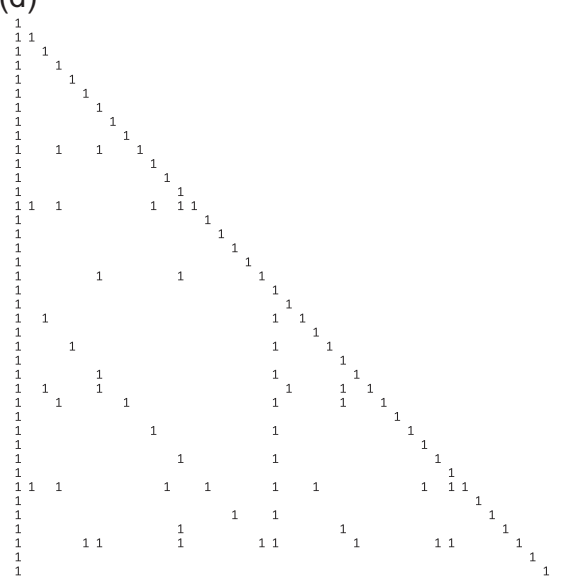

(b)

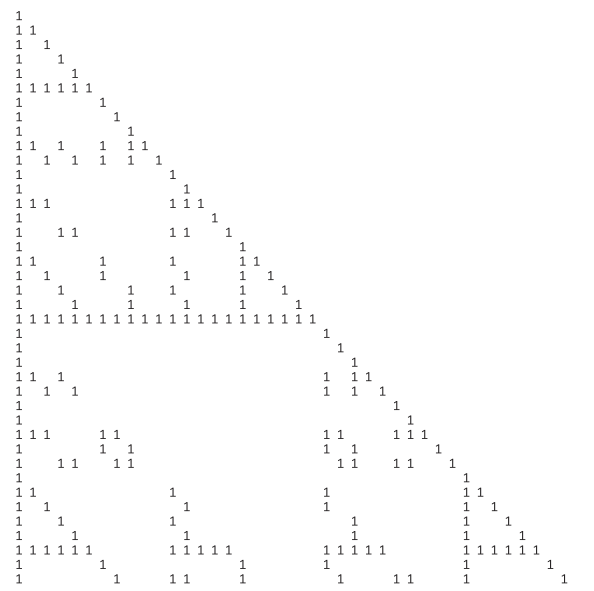

(e)

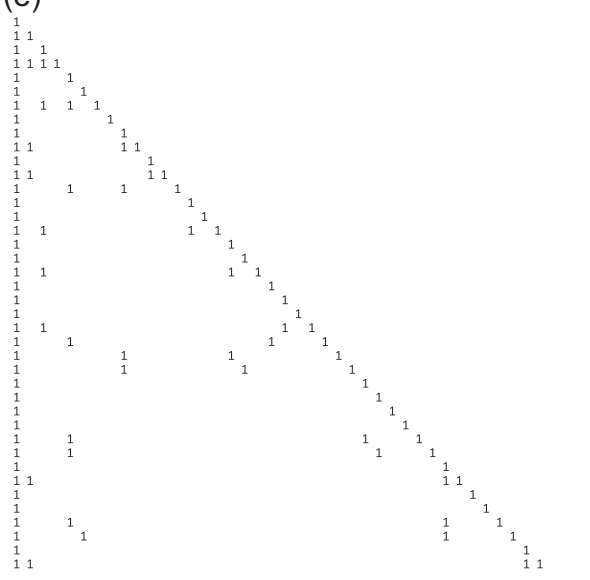

(c)

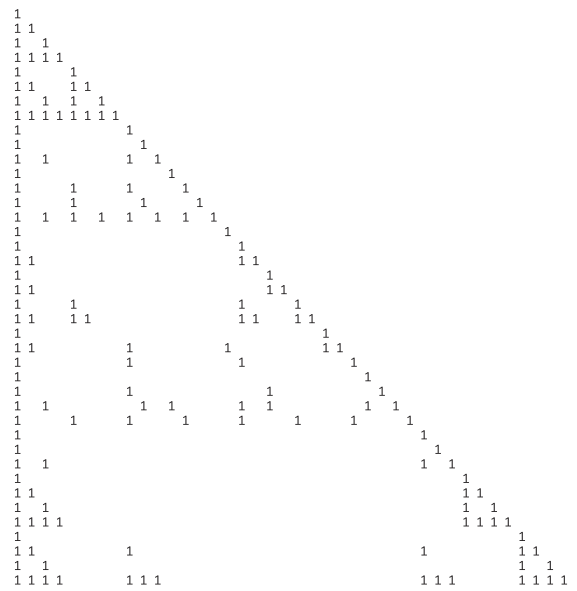

(f)

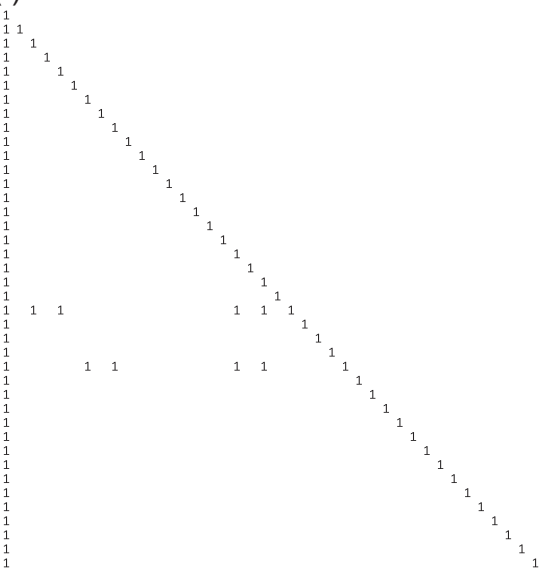

FIG. 7. Incommensurate RG of the Sierpinski triangle model. Spins at $(i, j)=(0,0)(\bmod 3)$ are picked up. (a) The original. (b) $\lambda=3$. (c) $\lambda=9$. (d) $\lambda=27$. (e) $\lambda=81$. (f) $\lambda=243$.

that among four RG'ed ground states, only two of them are independent.

It turns out that presence of discrete scale symmetries is a general property of classical Hamiltonians with interaction terms $Z(\bar{\alpha})$ for an arbitrary polynomial $\alpha$ over $\mathbb{F}_{p}$ in any spatial dimensions. We denote $\mathrm{RG}$ functions as $\mathrm{RG}_{i j}(i, j=$ $0, \ldots, p-1)$ which pick up spins at $(x, y)$ where $x=i(\bmod$ $p)$ and $y=j(\bmod p)$. When the Hamiltonian consists of $Z(\alpha)$, a ground state $\psi$ satisfies $Z\left(x^{i} y^{j} \ldots \alpha\right) \psi=\psi$ for all $i, j, \ldots$ So, one has $Z(\gamma \alpha) \psi=\psi$ for arbitrary polynomial $\gamma$. For $\gamma=\alpha^{p-1}$, one has $Z\left(\alpha^{p}\right) \psi=\psi$. From Eq. (8), $Z\left(\alpha^{p}\right)$ has supports only on sites $(i, j, \ldots)=(0,0, \ldots)(\bmod p)$. So, one has $Z(\alpha) \mathrm{RG}_{00}(\psi)=\mathrm{RG}_{00}(\psi)$, and $\mathrm{RG}_{00}(\psi)$ is a ground state for a smaller system. Since $\Gamma$ can be represented as linear combination of $\mathrm{RG}_{i j}, \Gamma(\psi)$ is also a ground state.

For simplicity of discussion, we concentrate on twodimensional cases with $Z(\bar{\alpha})$ and $\alpha=1-f(x) y$. Let us represent a ground state as $\psi_{m}=\gamma(x) \mathbf{f}_{m}(x, y)$ where $L=p^{m}$ and $\mathbf{f}_{m}(x, y)$ is the polynomial representation of the fractal. Then, $\mathrm{RG}$ 'ed states $\mathrm{RG}_{i j}\left(\psi_{m}\right)$ are always a ground state of the Hamiltonian for $L=p^{m-1}$. In particular, a polynomial $\gamma^{\prime}(x)$ satisfying the following equation always exists:

$$
\mathrm{RG}_{i j}\left[\gamma(x) \mathbf{f}_{m}\right]=\gamma^{\prime}(x) \mathbf{f}_{m-1}
$$

Following, we look at several examples. For $f=1+x$ over $\mathbb{F}_{3}$, one has

$$
\psi(1)=\left[\begin{array}{lllllllll}
1 & 0 & 0 & 0 & 0 & 0 & 0 & 0 & 0 \\
1 & 1 & 0 & 0 & 0 & 0 & 0 & 0 & 0 \\
1 & 2 & 1 & 0 & 0 & 0 & 0 & 0 & 0 \\
1 & 0 & 0 & 1 & 0 & 0 & 0 & 0 & 0 \\
1 & 1 & 0 & 1 & 1 & 0 & 0 & 0 & 0 \\
1 & 2 & 1 & 1 & 2 & 1 & 0 & 0 & 0 \\
1 & 0 & 0 & 2 & 0 & 0 & 1 & 0 & 0 \\
1 & 1 & 0 & 2 & 2 & 0 & 1 & 1 & 0 \\
1 & 2 & 1 & 2 & 1 & 2 & 1 & 2 & 1
\end{array}\right]
$$

and

$\mathrm{RG}_{0,0}(\psi(1))=\left[\begin{array}{ccc}1 & 0 & 0 \\ 1 & 1 & 0 \\ 1 & 2 & 1\end{array}\right], \quad \mathrm{RG}_{1,2}(\psi(1))=\left[\begin{array}{lll}2 & 0 & 0 \\ 2 & 2 & 0 \\ 2 & 1 & 2\end{array}\right]$. 
For the Fibonacci model $f=1+x+x^{2}$ over $\mathbb{F}_{2}$, one has

$$
\psi(1)=\left[\begin{array}{cccccccccc}
1 & 0 & 0 & 0 & 0 & 0 & 0 & 0 & 0 & \ldots \\
1 & 1 & 1 & 0 & 0 & 0 & 0 & 0 & 0 & \ldots \\
1 & 0 & 1 & 0 & 1 & 0 & 0 & 0 & 0 & \ldots \\
1 & 1 & 0 & 1 & 0 & 1 & 1 & 0 & 0 & \ldots \\
1 & 0 & 0 & 0 & 1 & 0 & 0 & 0 & 1 & \ldots \\
\vdots & \vdots & \vdots & \vdots & \vdots & \vdots & \vdots & \vdots & \vdots & \ddots
\end{array}\right] .
$$

RG'ed states are

$$
\mathrm{RG}_{00}(\psi(1))=\left[\begin{array}{cccccccccc}
1 & 0 & 0 & 0 & 0 & 0 & 0 & 0 & 0 & \ldots \\
1 & 1 & 1 & 0 & 0 & 0 & 0 & 0 & 0 & \ldots \\
1 & 0 & 1 & 0 & 1 & 0 & 0 & 0 & 0 & \ldots \\
1 & 1 & 0 & 1 & 0 & 1 & 1 & 0 & 0 & \ldots \\
1 & 0 & 0 & 0 & 1 & 0 & 0 & 0 & 1 & \ldots \\
\vdots & \vdots & \vdots & \vdots & \vdots & \vdots & \vdots & \vdots & \vdots & \ddots
\end{array}\right]
$$

and

$$
\mathrm{RG}_{01}(\psi(1))=\left[\begin{array}{cccccccccc}
1 & 1 & 0 & 0 & 0 & 0 & 0 & 0 & 0 & \ldots \\
1 & 0 & 0 & 1 & 0 & 0 & 0 & 0 & 0 & \ldots \\
1 & 1 & 1 & 1 & 1 & 1 & 0 & 0 & 0 & \ldots \\
1 & 0 & 1 & 1 & 1 & 1 & 0 & 1 & 0 & \ldots \\
1 & 1 & 0 & 0 & 1 & 1 & 0 & 0 & 1 & \ldots \\
\vdots & \vdots & \vdots & \vdots & \vdots & \vdots & \vdots & \vdots & \vdots & \ddots
\end{array}\right] .
$$

Discrete scale symmetries also arise at finite temperature as seen in distribution patterns of quasiparticle excitations. Consider an excited state with an excitation polynomial $E(x, y)$. An excitation energy $\Delta$ is given by the weight of excitation polynomial

$$
\Delta=2 W[E(x, y)]
$$

where $W[E(x, y)]$ counts the number of nonzero coefficients in $E(x, y)$. Let $\mathcal{D}_{\Delta}$ be a set of excitation polynomials with an excitation energy $\Delta$ :

$$
\mathcal{D}_{\Delta}=\{E(x, y): \Delta=2 W[E(x, y)]\} .
$$

Note

$$
E(x, y) \in \mathcal{D}_{\Delta} \Rightarrow E(x, y)^{p} \in \mathcal{D}_{\Delta},
$$

where $E(x, y)^{p}$ is a dilation of $E(x, y)$ by $p$. So, an excitation set $\mathcal{D}_{\Delta}$ is invariant under dilation by factor of $p$, and excitation pattern at finite temperature have discrete scale symmetries.

In condensed matter physics, one often encounters phase transition models governed by fixed points which exhibit dynamical scaling

$$
t \rightarrow \lambda^{z} t, \quad x \rightarrow \lambda x,
$$

where $z$ is called the dynamical scaling exponent. In conformally invariant systems, one always finds $z=1$. Examples of the anisotropic scale invariance with $z=2$ at the Lifshitz point often appears in condensed matter physics too. Classical fractal liquids correspond to cases with $z=0$ since excitation patterns have discrete scale symmetries for fixed energy although they are not at criticality.

\section{B. Limit cycles}

Discrete scale symmetries provide a useful algorithm to compute the fractal dimension of $\mathbf{f}(x, y)$. We illustrate the algorithm for the Fibonacci model: $f=1+x+x^{2}$ over $\mathbb{F}_{2}$. We denote a ground state with an initial condition $\gamma$ as $\psi(\gamma)$. Then, renormalization of ground states $\psi_{m}(1)$ and $\psi_{m}(1+x)$ gives the following ground states for $L=2^{m-1}$ :

$$
\begin{aligned}
& \mathrm{RG}_{00}\left[\psi_{m}(1)\right]=\psi_{m-1}(1), \quad \mathrm{RG}_{00}\left[\psi_{m}(1+x)\right]=\psi_{m-1}(1), \\
& \mathrm{RG}_{10}\left[\psi_{m}(1)\right]=\psi_{m-1}(0), \quad \mathrm{RG}_{10}\left[\psi_{m}(1+x)\right]=\psi_{m-1}(1), \\
& \mathrm{RG}_{01}\left[\psi_{m}(1)\right]=\psi_{m-1}(1+x), \quad \mathrm{RG}_{01}\left[\psi_{m}(1+x)\right]=\psi_{m-1}(1), \\
& \mathrm{RG}_{11}\left[\psi_{m}(1)\right]=\psi_{m-1}(1), \quad \mathrm{RG}_{11}\left[\psi_{m}(1+x)\right]=\psi_{m-1}(x) .
\end{aligned}
$$

Let us denote the weights of $\psi_{m}(1)$ and $\psi_{m}(1+x)$ as $A_{m}$ and $B_{m}$. Then, one has

$$
\left(\begin{array}{l}
A_{m} \\
B_{m}
\end{array}\right)=\left(\begin{array}{ll}
2 & 1 \\
4 & 0
\end{array}\right)\left(\begin{array}{l}
A_{m-1} \\
B_{m-1}
\end{array}\right) .
$$

This matrix has eigenvalues $1 \pm \sqrt{5}$ and, thus, $A_{m}$ and $B_{m}$ scale as $O\left(L^{\frac{\ln 1+\sqrt{5}}{\ln 2}}\right)$ for large $L$.

The above RG transformations concern classical fractal liquids on a finite lattice. If one performs RG transformations on an infinite lattice, $\mathrm{RG}_{i j}(\psi)$ becomes a group operation where $\mathrm{RG}_{i j}(\psi)$ is a linear map inside the ground-state space. In the case of a ferromagnet, the RG functions are always trivial; $\mathrm{RG}_{i j}(\psi)=\psi$ since $\psi$ is spatially uniform. Yet, for classical fractal liquids, $\mathrm{RG}_{i j}(\psi)$ may be different from $\psi$ in general.

This gives an interesting possibility of limit-cycle behaviors under RG transformations. Consider $f=1+x$ over $\mathbb{F}_{3}$. Let us apply a RG transformation for a ground state $\psi(1)=\mathbf{f}(x, y)$ where $\mathbf{f}(x, y)=1+f y+f^{2} y^{2}+\cdots$ is defined on an infinite lattice. Then, $\mathrm{RG}_{12}(\psi)$ gives the following sequence:

$$
\psi(1) \rightarrow \psi(2) \rightarrow \psi(1) \rightarrow \psi(2) \rightarrow \cdots,
$$

where a ground state $\psi(1)$ jumps to a different ground state $\psi(2)$, and the RG sequence exhibits a limit-cycle behavior. Next, for $f=1+x+x^{2}$ over $\mathbb{F}_{2}$, consider a ground state $\psi(1)=\mathbf{f}(x, y)$. Then, one has the following sequence under $\mathrm{RG}_{01}$ :

$$
\psi(1) \rightarrow \psi(1+x) \rightarrow \psi(1) \rightarrow \psi(1+x) \rightarrow \cdots,
$$

which is also a limit cycle.

Finally, consider $f=1+x+x^{2}$ over $\mathbb{F}_{5}$. We list some of its ground states as follows:

$$
\begin{aligned}
& {\left[\begin{array}{lllllllllll}
1 & & & & & & & & & \\
1 & 1 & 1 & & & & & & & \\
1 & 2 & 3 & 2 & 1 & & & & & \\
1 & 3 & 1 & 2 & 1 & 3 & 1 & & & \\
1 & 4 & 0 & 1 & 4 & 1 & 0 & 4 & 1 &
\end{array}\right],} \\
& {\left[\begin{array}{lllllllllll}
1 & 1 & & & & & & & & \\
1 & 2 & 2 & 1 & & & & & & \\
1 & 3 & 0 & 0 & 3 & 1 & & & & \\
1 & 4 & 4 & 3 & 3 & 4 & 4 & 1 & & \\
1 & 0 & 4 & 1 & 0 & 0 & 1 & 4 & 0 & 1
\end{array}\right],}
\end{aligned}
$$


(a)

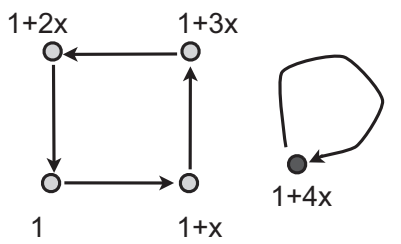

(b)

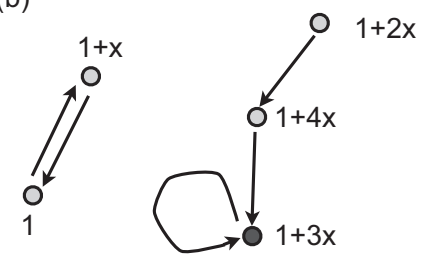

FIG. 8. Limit cycles in RG transformations for a classical fractal liquid with $f=1+x+x^{2}$ over $\mathbb{F}_{5}$. (a) $\mathrm{RG}_{02}$. (b) $\mathrm{RG}_{04}$.

$$
\begin{aligned}
& {\left[\begin{array}{lllllllllll}
1 & 2 & & & & & & & & \\
1 & 3 & 3 & 2 & & & & & & \\
1 & 4 & 2 & 3 & 0 & 2 & & & & \\
1 & 0 & 2 & 4 & 0 & 0 & 2 & 2 & & \\
1 & 1 & 3 & 1 & 1 & 4 & 2 & 4 & 4 & 2
\end{array}\right],} \\
& {\left[\begin{array}{rrrrrrrrrrr}
1 & 3 & & & & & & & & \\
1 & 4 & 4 & 3 & & & & & & \\
1 & 0 & 4 & 1 & 2 & 3 & & & & \\
1 & 1 & 0 & 0 & 2 & 1 & 0 & 3 & & \\
1 & 2 & 2 & 1 & 2 & 3 & 3 & 4 & 3 & 3
\end{array}\right],} \\
& {\left[\begin{array}{llllllllll}
1 & 4 & & & & & & & & \\
1 & 0 & 0 & 4 & & & & & & \\
1 & 1 & 1 & 4 & 4 & 4 & & & & \\
1 & 2 & 3 & 2 & 4 & 2 & 3 & 4 & & \\
1 & 3 & 1 & 2 & 4 & 3 & 4 & 4 & 2 & 4
\end{array}\right] .}
\end{aligned}
$$

$\mathrm{RG}_{02}$ generates the following limit cycle and fixed point:

$1 \rightarrow 1+x \rightarrow 1+3 x \rightarrow 1+2 x \rightarrow 1,1+4 x \rightarrow 1+4 x$,

where ground states are represented by $\gamma$ 's. A transformation $\mathrm{RG}_{04}$ leads to

$$
1 \rightarrow 1+x \rightarrow 1, \quad 1+2 x \rightarrow 1+4 x \rightarrow 1+3 x \rightarrow 1+3 x .
$$

These sequences are shown in Fig. 8. One may define renormalization function $\Gamma$ so that these fixed points and limit cycles are stable attractors.

\section{QUANTUM FRACTAL LIQUID}

\section{A. $\mathbb{Z}_{2}$ spin liquid and polynomial}

In this section, we present a general framework to construct a family of quantum fractal liquids which are a condensation of fractal objects. We begin by representing the $\mathbb{Z}_{2}$ spin liquid (the toric code) by polynomials. Following Ref. 36, we group two qubits into a single composite particle (Fig. 9) such that composite particles live on vertices of a square lattice:

$$
\begin{gathered}
S_{i, j}^{(Z)}=\left(Z_{A}\right)_{i, j}\left(Z_{B}\right)_{i, j}\left(Z_{A}\right)_{i+1, j}\left(Z_{B}\right)_{i, j+1}, \\
S_{i, j}^{(X)}=\left(X_{A}\right)_{i, j}\left(X_{B}\right)_{i, j}\left(X_{B}\right)_{i-1, j}\left(X_{A}\right)_{i, j-1},
\end{gathered}
$$

where each qubit inside a composite particle is labeled by $A$ and $B$. In polynomial representation, the parent Hamiltonian is

$$
H=-\sum_{i j} Z\left(\begin{array}{l}
x^{i} y^{j}(1+x) \\
x^{i} y^{j}(1+y)
\end{array}\right)-\sum_{i j} X\left(\begin{array}{c}
x^{i} y^{j}\left(1+y^{-1}\right) \\
x^{i} y^{j}\left(1+x^{-1}\right)
\end{array}\right)
$$
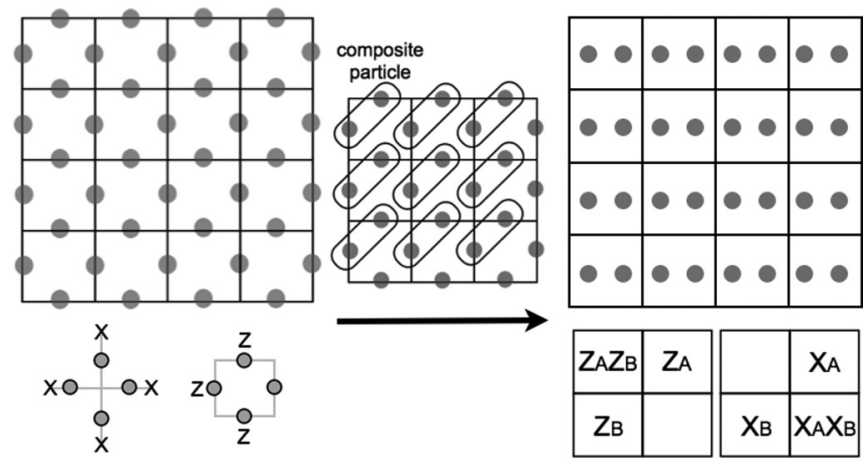

FIG. 9. Reduction of $\mathbb{Z}_{2}$ spin liquid. Two qubits are grouped into a composite particle which live inside each square.

where the upper (lower) row represents Pauli operators acting on $A(B)$. Interaction terms are translations of

$$
Z\left(\begin{array}{l}
1+x \\
1+y
\end{array}\right), \quad X\left(\begin{array}{c}
1+y^{-1} \\
1+x^{-1}
\end{array}\right) .
$$

In this form, it is immediate to see that the $\mathbb{Z}_{2}$ spin liquid consists of a pair of one-dimensional ferromagnets $Z(1+x)$ and $Z(1+y)$ at its one-dimensional limits.

Logical operators are

$$
\begin{aligned}
& \ell_{0}^{(Z)}=Z\left(\begin{array}{c}
0 \\
1+x+x^{2}+\cdots
\end{array}\right), \\
& \ell_{1}^{(Z)}=Z\left(\begin{array}{c}
1+y+y^{2}+\cdots \\
0
\end{array}\right), \\
& \ell_{0}^{(X)}=X\left(\begin{array}{c}
0 \\
1+y+y^{2}+\cdots
\end{array}\right), \\
& \ell_{1}^{(X)}=X\left(\begin{array}{c}
1+x+x^{2}+\cdots \\
0
\end{array}\right) .
\end{aligned}
$$

It is worth representing them graphically as follows:

$$
\begin{aligned}
\ell_{0}^{(Z)}= & {\left[\begin{array}{ccccc}
Z_{B} & Z_{B} & \ldots & Z_{B} & Z_{B} \\
I & I & \ldots & I & I \\
\vdots & \vdots & \ddots & \vdots & \vdots \\
I & I & \ldots & I & I \\
I & I & \ldots & I & I
\end{array}\right], } \\
\ell_{0}^{(Z)}= & {\left[\begin{array}{ccccc}
Z_{A} & I & I & \ldots & I \\
Z_{A} & I & I & \ldots & I \\
\vdots & \vdots & \vdots & \ddots & I \\
Z_{A} & I & I & \ldots & I \\
Z_{A} & I & I & \ldots & I
\end{array}\right] }
\end{aligned}
$$

and

$$
\ell_{0}^{(X)}=\left[\begin{array}{ccccc}
X_{B} & I & I & \ldots & I \\
X_{B} & I & I & \ldots & I \\
\vdots & \vdots & \vdots & \ddots & I \\
X_{B} & I & I & \ldots & I \\
X_{B} & I & I & \ldots & I
\end{array}\right],
$$




$$
\ell_{1}^{(X)}=\left[\begin{array}{ccccc}
X_{A} & X_{A} & \ldots & X_{A} & X_{A} \\
I & I & \ldots & I & I \\
\vdots & \vdots & \ddots & \vdots & \vdots \\
I & I & \ldots & I & I \\
I & I & \ldots & I & I
\end{array}\right] .
$$

One can see that logical operators commute with interaction terms by computing commutation polynomials.

One can generalize construction of $\mathbb{Z}_{2}$ spin liquid. For arbitrary polynomials $\alpha(x, y, \ldots)$ and $\beta(x, y, \ldots)$, consider

$$
Z\left(\begin{array}{c}
\alpha \\
\beta
\end{array}\right), \quad X\left(\begin{array}{c}
\bar{\beta} \\
\bar{\alpha}
\end{array}\right),
$$

where $\bar{\alpha}$ and $\bar{\beta}$ are duals of $\alpha$ and $\beta$ obtained by taking $x \rightarrow$ $x^{-1}, y \rightarrow y^{-1}, \ldots$. Note that interaction terms commute with each other as their commutation polynomial is $\alpha \beta+\beta \alpha=0$ over $\mathbb{F}_{2}$. A parent Hamiltonian is

$$
H=-\sum_{i j \ldots} Z\left(\begin{array}{l}
x^{i} y^{j} \ldots \alpha \\
x^{i} y^{j} \ldots \beta
\end{array}\right)-\sum_{i j \ldots} X\left(\begin{array}{c}
x^{i} y^{j} \ldots \bar{\beta} \\
x^{i} y^{j} \ldots \bar{\alpha}
\end{array}\right) .
$$

As for generalization to $\mathbb{F}_{p}$, we take $Z(\alpha, \beta)^{T}$ and $X(-\bar{\beta}, \bar{\alpha})$ so that the commutation polynomial is $\alpha(-\beta)+\beta \alpha=0$.

\section{B. Quantum fractal liquid}

Consider a three-dimensional $L \times L \times L$ square lattice where two qubits live on each site with $L=2^{m}$ and periodic boundary conditions. Quantum fractal liquids have

$$
\alpha=1-f(x) y, \quad \beta=1-g(x) z
$$

in Eq. (24) where $f(x)$ and $g(x)$ are reversible polynomials over $\mathbb{F}_{2}$. More explicitly, interaction terms are translations of

$$
Z\left(\begin{array}{c}
1-f(x) y \\
1-g(x) z
\end{array}\right), \quad X\left(\begin{array}{c}
1-\bar{g}(x) \bar{z} \\
1-\bar{f}(x) \bar{y}
\end{array}\right) .
$$

Interaction terms are characterized by a pair of fractal models $Z[1-f(x) y]$ and $Z[1-g(x) z]$. In this sense, quantum fractal liquids can be viewed as a coherent combination of a pair of classical fractal liquids living on the $(\hat{x}, \hat{y})$ and $(\hat{x}, \hat{z})$ planes, respectively.

Logical operators of quantum fractal liquids have fractal shapes which are generated by polynomials $f(x)$ and $g(x)$ :

$$
\begin{aligned}
& \mathbf{f}(x, y)=1+f y+f^{2} y^{2}+\cdots, \\
& \overline{\mathbf{f}}(x, y)=1+\bar{f} \bar{y}+\bar{f}^{2} \bar{y}^{2}+\cdots, \\
& \mathbf{g}(x, z)=1+g z+g^{2} z^{2}+\cdots, \\
& \overline{\mathbf{g}}(x, y)=1+\bar{g} \bar{z}+\bar{g}^{2} \bar{z}^{2}+\cdots,
\end{aligned}
$$

Note $\mathbf{f}(x, y)$ lives on a $(\hat{x}, \hat{y})$ plane while $\mathbf{g}(x, z)$ lives on a $(\hat{x}, \hat{z})$ plane. Quantum fractal liquids have $k=2 L$, and there are $2 L$ of $Z$-type logical operators and $2 L$ of $X$-type logical operators:

$$
\begin{aligned}
& \ell_{i}^{(Z)}=Z\left(\begin{array}{c}
0 \\
x^{i} \mathbf{f}(x, y)
\end{array}\right), \quad r_{i}^{(Z)}=Z\left(\begin{array}{c}
x^{i} \mathbf{g}(x, z) \\
0
\end{array}\right), \\
& \ell_{i}^{(X)}=X\left(\begin{array}{c}
x^{i} \mathbf{\mathbf { f }}(x, y) \\
0
\end{array}\right), \quad r_{i}^{(X)}=X\left(\begin{array}{c}
0 \\
x^{i} \overline{\mathbf{g}}(x, z)
\end{array}\right),
\end{aligned}
$$

where $i=0, \ldots, L-1$. Therefore, $Z$-type logical operators have geometric shapes of $\mathbf{f}(x, y)$ and $\mathbf{g}(x, y)$, while $X$-type logical operators have geometric shapes of $\overline{\mathbf{f}}(x, y)$ and $\overline{\mathbf{g}}(x, y)$.

To show that the above operators are logical operators, we need to verify the following two things: (a) they commute with interaction terms, and (b) they can be grouped into pairs of anticommuting logical operators. One may see that logical operators commute with all the interaction terms by computing commutation polynomials. For instance, a commutation polynomial between $\ell_{0}^{(Z)}=Z(0, \mathbf{f}(x, y))^{T}$ and stabilizers $X(1-\bar{g} \bar{y}, 1-\bar{f} \bar{x})^{T}$ is given by $(1-f y) \mathbf{f}(x, y)=$ 0 for reversible $f$. Logical operators obey the following commutation relations:

$$
\left\{\begin{array}{cc}
\ell_{0}^{(Z)}, \ldots, \ell_{L-1}^{(Z)}, & r_{0}^{(Z)}, \ldots, r_{L-1}^{(Z)} \\
r_{0}^{(X)}, \ldots, r_{L-1}^{(X)}, & \ell_{0}^{(X)}, \ldots, \ell_{L-1}^{(X)}
\end{array}\right\} .
$$

Generalization to $\mathbb{F}_{p}$ is also possible.

To see that quantum fractal liquids are topologically ordered, we begin by showing that they are good quantum error-correcting codes with $d \rightarrow \infty$ for $L \rightarrow \infty$ where $d$ is the quantum code distance of the ground-state space. A standard way to prove this considers a bipartition of the system into two complementary subsets $A$ and $B$ and uses the following bipartition formula which holds for arbitrary stabilizer codes $:^{38}$

$$
g_{A}+g_{B}=2 k,
$$

where $g_{A}$ and $g_{B}$ represent the number of independent logical operators supported inside $A$ and $B$, respectively. Let us assume $A$ to be a connected region with finite support. Then, its complementary subset $B$ accommodates some $(\hat{x}, \hat{y})$ plane and $(\hat{x}, \hat{z})$ plane where all the $2 k$ independent logical operators can be supported. So, one has $g_{B}=2 k$. This leads to $g_{A}=0$. Therefore, weights of logical operators are not finite (i.e., unbounded), and $d \rightarrow \infty$ for $L \rightarrow \infty$.

For stabilizer Hamiltonians, being a quantum code $(d \rightarrow$ $\infty$ for $L \rightarrow \infty$ ) automatically implies the presence of topological order with stability against local perturbations. Bravyi, Hastings, and Michalakis ${ }^{25}$ proved that frustration-free Hamiltonians with an ability of quantum error-correcting code have stability against local perturbations when Hamiltonians satisfy a certain condition, called TQO-2. Roughly speaking, TQO-2 states that locally computed density matrices are consistent with ground states which are computed globally. One can check that quantum fractal liquids satisfy TQO2 by explicit calculations, and thus have stability against local perturbations. Recall that quantum fractal liquids have $2^{2 L}$ ground states. Under sufficiently small but finite local perturbations, the energy splitting among these ground states is always exponentially suppressed, and the energy gap between the ground states and excited states remains finite.

We then discuss the number of degenerate ground states and their dependence on the system size. A key feature of quantum fractal liquids is that the number of logical qubits $k$ has a fairly sensitive dependence on the system size $\vec{L}=$ $\left(L_{1}, L_{2}, L_{3}\right)$. It turns out that the number of logical qubits $k$ is given by counting the dimension of solutions $\gamma$ satisfying the following equation:

$$
f(x)^{L_{2}} \gamma(x)=g(x)^{L_{3}} \gamma(x)=\gamma(x), \quad x^{L_{1}}=1
$$


with $k=2 \operatorname{dim} \gamma$. For instance, with $f=x^{i}$ and $g=x^{j}$, one has $k=2 \operatorname{gcd}\left(L_{1}, i L_{2}, j L_{3}\right)$ where $k$ depends crucially on the system size $\vec{L}=\left(L_{1}, L_{2}, L_{3}\right)$. In general, it is a very challenging task to write an explicit form of $k\left(L_{1}, L_{2}, L_{3}\right)$ for a given pair of $f(x)$ and $g(x)$. Yet, $k\left(L_{1}, L_{2}, L_{3}\right)$ has a nice symmetry property under scale transformations:

$$
k\left(p L_{1}, p L_{2}, p L_{3}\right)=p k\left(L_{1}, L_{2}, L_{3}\right) .
$$

This can be proven from discrete scale symmetries of polynomials over $\mathbb{F}_{p}$.

Ground states of quantum fractal liquids correspond to limit cycles under real-space RG transformations on an infinite lattice. To obtain RG transformations with meaningful attractors that do not flow to disordered states or trivial product states, one needs to apply some appropriate projection operators on sites that are to be coarse grained. Following, we present an example of such projections. Consider a pair of qubits at site $(i, j, \ell)$, denoted as $|\phi\rangle_{i j \ell}=\left|\phi_{A}\right\rangle_{i j \ell} \otimes\left|\phi_{B}\right\rangle_{i j \ell}$, and apply the following projections to a ground state:

$$
\left(I+Z_{A}^{\ell} \otimes Z_{B}^{j}\right)\left(I+X_{A}^{j} \otimes X_{B}^{\ell}\right)\left|\phi_{A}\right\rangle_{i j \ell} \otimes\left|\phi_{B}\right\rangle_{i j \ell} .
$$

Note that projection operators commute with each other, and projections are applied only to sites $(i, j, \ell)$ with $j \neq 0$ or $\ell \neq 0$ modulo 2. As a result, pairs of qubits on sites $(i, j, \ell)$ with $j=\ell=0$ modulo 2 are completely decoupled from the rest. With some calculations, one notices that stabilizer generators for remaining sites $(i, j, \ell)$ with $j=\ell=0$ modulo 2 are given by

$$
Z\left(\begin{array}{c}
\alpha^{2} \\
\beta^{2}
\end{array}\right), \quad Z\left(\begin{array}{c}
x \alpha^{2} \\
x \beta^{2}
\end{array}\right), \quad X\left(\begin{array}{c}
\bar{\beta}^{2} \\
\bar{\alpha}^{2}
\end{array}\right), \quad X\left(\begin{array}{c}
x \bar{\beta}^{2} \\
x \bar{\alpha}^{2}
\end{array}\right),
$$

and their translations that are generated by applications of $x^{2 i^{\prime}} y^{2 j^{\prime}} z^{2 \ell^{\prime}}$. (See Ref. 39 for transformations of stabilizer generators under projections.) This corresponds to two copies of original quantum fractal liquids. Let us pick up sites with $(i, j, \ell)=(0,0,0)$ modulo 2 and throw away sites with $(i, j, \ell)=$ $(1,0,0)$ modulo 2 via some arbitrary projections. Rescaling by $x^{2} \rightarrow x, y^{2} \rightarrow y$, and $z^{2} \rightarrow z$, stabilizer generators are $Z(\alpha, \beta)^{T}$ and $X(\bar{\beta}, \bar{\alpha})^{T}$. So, this RG transformation maps a ground state of a quantum fractal liquid into some ground state which may be different from the original. One can keep track of how ground states flow under RG transformations by looking at polynomial representation of fractal logical operators. This can be analyzed in exactly the same way as ground states of classical fractal liquids.

\section{QUASIPARTICLES}

In this section, we discuss quasiparticle excitations and derive a necessary and sufficient condition for quantum fractal liquids to be free from stringlike logical operators. Several examples of quantum fractal liquids are also studied, and the cubic code is shown to be unitarily equivalent to a model of second-order quantum fractal liquid.

\section{A. Criteria for no string}

We discuss properties of quasiparticle excitations in quantum fractal liquids. Without loss of generality, one can
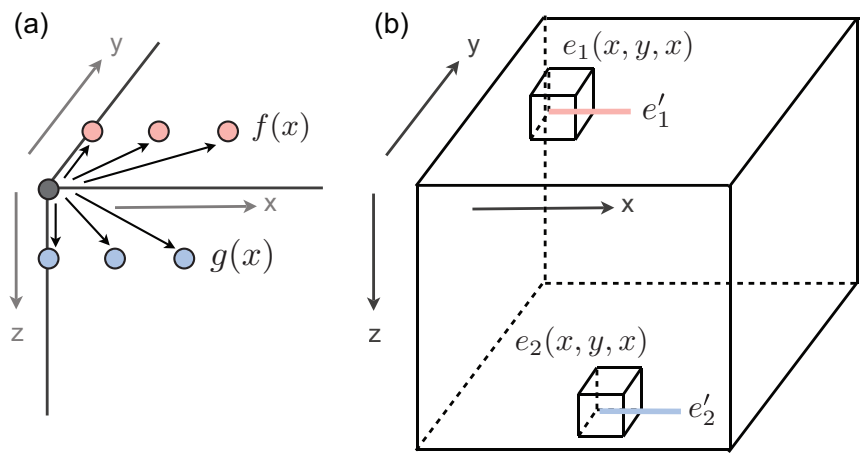

FIG. 10. (Color online) (a) Propagation of quasiparticles by $f(x)$ and $g(x)$. (b) A pair of localized excitations $e_{1}$ and $e_{2}$ with elongated excitations $e_{1}^{*}$ and $e_{2}^{*}$.

concentrate on excitations caused by Pauli-Z-type operators (phase errors) which flip $X$-type interaction terms. Following a treatment of classical fractal liquids, we represent positions of excitations as an excitation polynomial

$$
E(x, y, z)=\sum_{i, j, \ell} c_{i j \ell} x^{i} y^{j} z^{\ell} \quad \text { over } \mathbb{F}_{p},
$$

where $c_{i j \ell}=1$ means an excitation is present at $(i, j, \ell)$. We consider excitations caused by a Pauli operator $Z\left(e_{A}, e_{B}\right)^{T}$. They are given by a commutation polynomial between $\left(e_{A}, e_{B}\right)^{T}$ and $(-\bar{\beta}, \bar{\alpha})^{T}$ :

$$
E(x, y, z)=-e_{A}(1-g z)+e_{B}(1-f y) .
$$

Excitations caused by a Pauli operator $Z_{0,0,0}^{(A)}\left(e_{A}=1, e_{B}=\right.$ $0)$ are given by

$$
E(x, y, z)=-(1-g z),
$$

while excitations caused by a Pauli operator $Z_{0,0,0}^{(B)}\left(e_{A}=\right.$ $\left.0, e_{B}=1\right)$ are given by

$$
E(x, y, z)=(1-f y) .
$$

So, if an isolated excitation is present at $(i, j, \ell)=(0,0,0)$, it will propagate to multiple excitations represented by $g z$ via an application of $Z_{0,0,0}^{(A)}$ [Fig. 10(a)]. Similarly, it will propagate to multiple excitations represented by $f y$ via an application of $\left[Z_{0,0,0}^{(B)}\right]^{-1}$ [Fig. 10(a)]. In general, for a single isolated excitation, $f(x)$ is applied when propagating in the $\hat{y}$ direction, and $g(x)$ is applied when propagating in the $\hat{z}$ direction.

An analogy to cellular automaton becomes transparent by considering propagation of a one-dimensional excitation pattern $e(x)$, located at $j=\ell=0$. It will propagate in the $\hat{y}$ and $\hat{z}$ directions as follows:

$$
E(x, y, z)=e(x) f(x)^{j^{\prime}} g(x)^{\ell^{\prime}} y^{j^{\prime}} z^{\ell^{\prime}} .
$$

This may be viewed as time evolution of an initial condition $e(x)$, updated $j^{\prime}$ times by $f(x)$ and $\ell^{\prime}$ times by $g(x)$, respectively.

We first assume $f(x)=g(x)$, and consider propagation of $e(x)$ :

$$
E(x, y, z)=f(x)^{j^{\prime}+\ell^{\prime}} y^{j^{\prime}} z^{\ell^{\prime}}
$$


For $j^{\prime}+\ell^{\prime}=0$, excitations are single quasiparticles, and excitation energy remains finite. Therefore, quasiparticle excitations can propagate freely (without costing much energy) in the $\hat{y}-\hat{z}$ direction. This implies the presence of a stringlike logical operator. Indeed, the following stringlike operators are logical operators:

$$
Z\left(\begin{array}{c}
(y+z)^{L-1} \\
(y+z)^{L-1}
\end{array}\right), \quad X\left(\begin{array}{c}
(\bar{y}+\bar{z})^{L-1} \\
(\bar{y}+\bar{z})^{L-1}
\end{array}\right) .
$$

Note that $(y+z)^{L-1}$ is a stringlike polynomial extending in the $\hat{y}-\hat{z}$ direction.

When do we have stringlike logical operators? Without loss of generality, one can assume that $f(x)$ and $g(x)$ start from the origin, meaning that $f(x)$ and $g(x)$ have nonzero constant terms and have only positive powers. (Otherwise, we shift lattice positions.) We say that $f(x)$ and $g(x)$ are algebraically related when there exists some finite integers $c_{f}$ and $c_{g}$ such that

$$
f(x)^{c_{f}}=\text { const } \times g(x)^{c_{g}}
$$

without considering periodic boundary conditions. Then, one notices that quasiparticles can propagate in the $c_{a} \hat{y}-c_{b} \hat{z}$ direction, and there exist stringlike logical operators.

It turns out that Eq. (35) is a necessary and sufficient condition for the presence of stringlike logical operators:

\section{No stringlike logical operator}

$$
\Leftrightarrow f(x) \text { and } g(x) \text { are not algebraically related. }
$$

Our argument follows Ref. 40. Let us assume that a stringlike logical operator exists. By taking a subpart of stringlike logical operator, we may consider a pair of localized excitations $e_{1}(x, y, x)$ and $e_{2}(x, y, x)$ that are created at end points of stringlike logical operators. We assume that excitations are contained in two cubic boxes of $w \times w \times w$ sites which are separated by $L_{\delta}$ with $L \gg L_{\delta} \gg w$.

One can make quasiparticle excitations in $e_{1}(x, y, z)$ and $e_{2}(x, y, z)$ propagate by applying $f(x)$ and $g(x)$ such that they become elongated one-dimensional excitations whose lengths are $O(w)$ [see Fig. 10(b)]. We denote polynomials corresponding to these elongated one-dimensional excitations as $e_{1}^{\prime}$ and $e_{2}^{\prime}$, and assume that $e_{1}^{\prime}$ is at $j=\ell=0$ and $e_{2}^{\prime}$ is at $j=j^{\prime}$ and $\ell=\ell^{\prime}$ where $\left|j^{\prime}\right|+\left|\ell^{\prime}\right| \sim O\left(L_{\delta}\right)$. One may write $e_{1}^{\prime}=e_{1}^{*}(x)$ and $e_{2}^{\prime}=e_{2}^{*}(x) y^{j^{\prime}} z^{\ell^{\prime}}$. Then, one must have $e_{2}^{*}=-e_{1}^{*} f(x)^{j^{\prime}} g(x)^{\ell^{\prime}}$ as one can eliminate them by making $e_{1}^{\prime}$ propagate and collide with $e_{2}^{\prime}$.

Since a pair of excitations is created by a stringlike object (a subpart of stringlike logical operator) with finite width, $f^{j^{\prime}} g^{\ell^{\prime}}$ must remain finite for large $j^{\prime}$ and $\ell^{\prime}$. This requires $f$ and $g$ to be algebraically related; otherwise, the size of $f^{j^{\prime}} g^{\ell^{\prime}}$ grows at least linearly as $\left|j^{\prime}\right|$ and $\left|\ell^{\prime}\right|$ grow. Therefore, the presence of stringlike logical operators implies Eq. (35).

\section{B. Several examples}

Here, we study several examples of quantum fractal liquids. (a) We begin with a trivial case with $f=g=1$ :

$$
Z\left(\begin{array}{l}
1+y \\
1+z
\end{array}\right) \text { over } \mathbb{F}_{2} .
$$

This is a stack of slices of two-dimensional $\mathbb{Z}_{2}$ model where each copy lives on a $(\hat{y}, \hat{z})$ plane. It has pairs of stringlike logical operators since $f=1$ and $g=1$ are generators of strings. Similarly, for $f=1$ and $g=1$ over $\mathbb{F}_{p}(p>2)$, the model is a stack of $\mathbb{Z}_{p}$ spin liquids with $p$-dimensional spins. by

(b) For $f=x$ and $g=1$, stabilizer generators are given

$$
Z\left(\begin{array}{c}
1+x y \\
1+z
\end{array}\right) \text { over } \mathbb{F}_{2}
$$

This is a stack of slices of two-dimensional $\mathbb{Z}_{2}$ model, but each copy lives on a $(\hat{x}+\hat{y}, \hat{z})$ plane. It has stringlike logical operators, running in the $\hat{x}+\hat{y}$ direction. One can reduce this model to the model in (a) by a modular transformation $x \rightarrow x$, $x y \rightarrow y$, and $z \rightarrow z$ which corresponds to a lattice distortion.

(c) Let us look at an example with pairs of fractal logical operators and stringlike logical operators:

$$
Z\left(\begin{array}{c}
1+\left(1+x+x^{2}\right) y \\
1+z
\end{array}\right) \text { over } \mathbb{F}_{2}
$$

Geometric shapes of fractal logical operators are generated by $f=1+x+x^{2}$ (the Fibonacci model). Fractal logical operators live on a two-dimensional plane and stringlike logical operators penetrate the two-dimensional plane.

(d) Some models do not have any logical qubits under periodic boundary conditions:

$$
Z\left(\begin{array}{c}
1+(1+x) y \\
1+z
\end{array}\right) \text { over } \mathbb{F}_{2}
$$

since $f=1+x$ is not reversible over $\mathbb{F}_{2}$. When the model is defined with open boundary conditions, it has pairs of Sierpinski-type logical operators and stringlike logical operators.

(e) Consider

$$
Z\left(\begin{array}{c}
1+(1+x) y \\
1+(1+x) z
\end{array}\right) \text { over } \mathbb{F}_{2}
$$

The model has pairs of fractal logical operators, but has hidden stringlike logical operators running in the $\hat{y}-\hat{z}$ direction. In fact, this model is unitarily equivalent to the following model:

$$
Z\left(\begin{array}{c}
1+(1+x) y \\
1+y^{-1} z
\end{array}\right) \text { over } \mathbb{F}_{2} \text {. }
$$

(f) Let us consider the cases without any stringlike logical operators for $\mathrm{F}_{2}$ :

$$
Z\left(\begin{array}{l}
1+\left(1+x+x^{2}\right) y \\
1+\left(1+x+x^{3}\right) z
\end{array}\right) \text { over } \mathbb{F}_{2}
$$

The model is free from stringlike logical operators as $f=$ $1+x+x^{2}$ and $g=1+x+x^{3}$ are algebraically unrelated over $\mathbb{F}_{2}$. Interaction terms are seven body. 
(g) Next, let us consider a model over $\mathbb{F}_{p}(p>2)$ :

$$
Z\left(\begin{array}{c}
1+(1+x) y \\
1+\left(1+x^{2}\right) z
\end{array}\right) \text { over } \mathbb{F}_{3} .
$$

The model is also free from stringlike logical operators as $1+$ $x$ and $1+x^{2}$ are algebraically unrelated over $\mathbb{F}_{3}$. Interaction terms are five body.

(h) The cubic code can be viewed as a second-order quantum fractal liquid. In polynomial representation, one has

$$
Z\left(\begin{array}{c}
1+x+y+z \\
1+x y+y z+z x
\end{array}\right) \text { over } \mathbb{F}_{2}
$$

The model can be mapped to the following second-order quantum fractal liquid through local unitary transformations and modular transformations:

$$
Z\left(\begin{array}{c}
1+f(x) y \\
1+g_{1}(x) z+g_{2}(x) z^{2}
\end{array}\right) \text { over } \mathbb{F}_{2},
$$

where

$f(x)=1+x+x^{2}, \quad g_{1}(x)=1+x, \quad g_{2}(x)=1+x+x^{2}$.

Two-qubit gates can be characterized by a two-qubit Pauli operator $V=V_{1} \otimes V_{2}$. Consider an arbitrary two-qubit Pauli operator $U=U_{1} \otimes U_{2}$. A two-qubit gate generated by $V$ transforms $U$ as follows:

$$
U_{1} \otimes U_{2} \rightarrow U_{1} V_{1}^{c_{2}} \otimes U_{2} V_{2}^{c_{1}},
$$

where $U_{1} V_{1}=(-1)^{c_{1}} V_{1} U_{1}$ and $U_{2} V_{2}=(-1)^{c_{2}} V_{2} U_{2}$. For instance, with $V=X_{1} \otimes X_{2}$, one has the following transformations:

$$
\left\{\begin{array}{ll}
Z_{1}, & Z_{2} \\
X_{1}, & X_{2}
\end{array}\right\} \rightarrow\left\{\begin{array}{cc}
Z_{1} X_{2}, & X_{1} Z_{2} \\
X_{1}, & X_{2}
\end{array}\right\}
$$

These two-qubit gates may be viewed as generalizations of control- $Z$ operation. One may see that transformations in Eq. (36) preserve commutation relations by direct calculations, and thus are indeed unitary transformations.

Let us apply these two-qubit gates to a canonical model with $Z(\alpha, \beta)^{T}$ and $X(\bar{\beta}, \bar{\alpha})^{T}$. We think of applying a sequence of two-qubit gates, characterized by $X_{A} \otimes Z_{B}$, on neighboring sites in the $\hat{x}$ direction. Then, one has the following transformations:

$$
Z\left(\begin{array}{c}
\alpha \\
\beta
\end{array}\right) \rightarrow Z\left(\begin{array}{c}
\alpha \\
\beta+\alpha x
\end{array}\right), \quad X\left(\begin{array}{l}
\bar{\beta} \\
\bar{\alpha}
\end{array}\right) \rightarrow X\left(\begin{array}{c}
\bar{\beta}+\bar{\alpha} \bar{x} \\
\bar{\alpha}
\end{array}\right),
$$

which correspond to $\alpha \rightarrow \alpha$ and $\beta \rightarrow \beta+x \alpha$. Note that these two-qubit gates can be applied simultaneously as they commute with each other. By generalizing this transformation, the following transformations are allowed:

$$
\alpha \rightarrow \alpha, \quad \beta \rightarrow \beta+x^{i} y^{j} z^{\ell} \alpha,
$$

where $i, j, \ell$ are some finite integers.

For the cubic code $(\alpha=1+x+y+z, \beta=1+x y+$ $y z+z x)$, we apply two-qubit gates $(\alpha, \beta) \rightarrow(\alpha, \beta+x \alpha)$, modular transformations $(x, y, z) \rightarrow\left(x, y z^{-1}, z\right)$, shifting of lattice sites in the $\hat{z}$ direction, and two-qubit gates $(\alpha, \beta) \rightarrow$

$$
\begin{aligned}
& (\alpha+\beta, \beta): \\
& \left(\begin{array}{c}
1+x+y+z \\
1+x y+y z+z x
\end{array}\right) \rightarrow\left(\begin{array}{c}
1+x+y+z \\
1+x+x^{2}+y z
\end{array}\right) \\
& \quad \rightarrow\left(\begin{array}{c}
1+x+y z^{-1}+z \\
1+x+x^{2}+y
\end{array}\right) \rightarrow\left(\begin{array}{c}
y+(1+x) z+z^{2} \\
1+x+x^{2}+y
\end{array}\right) \\
& \quad \rightarrow\left(\begin{array}{c}
\left(1+x+x^{2}\right)+(1+x) z+z^{2} \\
1+x+x^{2}+y
\end{array}\right)
\end{aligned}
$$

\section{OUTLOOK}

In this paper, we have presented a general construction of classical and quantum fractal liquids and demonstrated that they have exotic physical properties beyond theory with continuous scale invariance. We hope that our construction and theory of fractal spin liquids will provide a stepping stone toward complete understanding and classification of quantum phases of matter.

Quasiparticle excitations arising in quantum fractal liquids are all Abelian. Topological order arising in quantum fractal liquids is nonchiral. It is unclear to what extent our results may generalize to chiral topological phases, non-Abelian topological phases, and symmetry-protected topological phases. Effective field theoretical descriptions of classical and quantum fractal liquids are currently not known. It may be interesting to analyze how classical and quantum fractal liquids behave under RG transformations in the language of matrix and tensor product state representations. ${ }^{41-44}$ An underlying difficulty in physically realizing the Sierpinski triangle model lies in the fact that the model has three-body interaction terms. Yet, one may simulate three-body interactions easily by using hopping particles. ${ }^{45}$ Construction of quantum fractal liquids builds on cellular automaton with linear update rules, and nonlinear extension remains as an open problem.

Another important motivation behind this paper concerns quantum information storage capacity of discrete spin systems. There is a well-known bound on the amount of quantum information that can be stored reliably in a given volume of discrete spin systems which are supported by gapped local Hamiltonians. ${ }^{46}$ However, all the previously known systems were far below this theoretical bound, and it remains open as to whether there exists a gapped spin system that saturates this bound. We have solved a classical version of this problem ${ }^{20}$ by proving that a family of Sierpinski-type classical fractal liquids asymptotically saturate the classical information storage capacity bound. With this success in hand, we hope that quantum fractal liquids, which are a natural generalization of classical fractal liquids, also asymptotically saturate the quantum information storage capacity bound. Analysis on coding properties of quantum fractal liquids is an important open problem which may lead to discovery of capacity saturating quantum error-correcting codes.

\section{ACKNOWLEDGMENTS}

I thank P. Shor, J. Preskill, X.-G. Wen, and S. Takayoshi for helpful discussion and comments. I acknowledge fruitful 
discussion with O. Buerschaper on RG transformations. I thank J. Haah, S. Michalakis, and A. Kubica for countless discussions at Caltech. I am supported by the David and Ellen Lee Postdoctoral fellowship. I acknowledge funding provided by the Institute for Quantum Information and Matter, an NSF Physics Frontiers Center with support of the Gordon and Betty Moore Foundation (Grants No. PHY-0803371 and No. PHY-1125565).
${ }^{1}$ X. G. Wen and Q. Niu, Phys. Rev. B 41, 9377 (1990).

${ }^{2}$ A. Y. Kitaev, Ann. Phys. (NY) 303, 2 (2003).

${ }^{3}$ D. C. Tsui, H. L. Stormer, and A. C. Gossard, Phys. Rev. Lett. 48, 1559 (1982).

${ }^{4}$ R. B. Laughlin, Phys. Rev. Lett. 50, 1395 (1983).

${ }^{5}$ X. G. Wen, F. Wilczek, and A. Zee, Phys. Rev. B 39, 11413 (1989).

${ }^{6}$ N. Read and S. Sachdev, Phys. Rev. Lett. 66, 1773 (1991).

${ }^{7}$ X. G. Wen, Phys. Rev. B 44, 2664 (1991).

${ }^{8}$ P. W. Anderson, Science 235, 1196 (1987).

${ }^{9}$ I. Affleck, T. Kennedy, E. H. Lieb, and H. Tasaki, Phys. Rev. Lett. 59, 799 (1987).

${ }^{10}$ T. Senthil and M. P. A. Fisher, Phys. Rev. B 62, 7850 (2000).

${ }^{11}$ R. Moessner and S. L. Sondhi, Phys. Rev. Lett. 86, 1881 (2001).

${ }^{12}$ A. Kitaev, Ann. Phys. (NY) 321, 2 (2006).

${ }^{13}$ E. Witten, Commun. Math. Phys. 117, 353 (1988).

${ }^{14}$ M. Atiyah, Publ. Math. I.H.E.S. 68, 175 (1988).

${ }^{15}$ M. A. Levin and X.-G. Wen, Phys. Rev. B 71, 045110 (2005).

${ }^{16}$ J. Haah, Phys. Rev. A 83, 042330 (2011).

${ }^{17}$ I. H. Kim, arXiv:1202.0052.

${ }^{18}$ C. Castelnovo and C. Chamon, Philos. Mag. 92, 304 (2012).

${ }^{19}$ M. E. J. Newman and C. Moore, Phys. Rev. E 60, 5068 (1999).

${ }^{20}$ B. Yoshida, Ann. Phys. (NY) 338, 134 (2013).

${ }^{21}$ C. Nayak, S. H. Simon, A. Stern, M. Freedman, and S. Das Sarma, Rev. Mod. Phys. 80, 1083 (2008).

${ }^{22}$ J. Haah, arXiv:1204.1063.

${ }^{23}$ C. Castelnovo, C. Chamon, and D. Sherrington, Phys. Rev. B 81, 184303 (2010).

${ }^{24}$ X.-G. Wen, Phys. Rev. Lett. 90, 016803 (2003).

${ }^{25}$ S. Bravyi, M. Hastings, and S. Michalakis, J. Math. Phys. 51, 093512 (2010).
${ }^{26}$ B. Yoshida, Ann. Phys. (NY) 326, 2566 (2011).

${ }^{27} \mathrm{~S}$. Wolfram, A New Kind of Science (Wolfram Media Inc., Champaign, 2002).

${ }^{28}$ A. P. Ramirez, Annu. Rev. Mater. Sci. 24, 453 (1994).

${ }^{29}$ M. J. Harris, S. T. Bramwell, D. F. McMorrow, T. Zeiske, and K. W. Godfrey, Phys. Rev. Lett. 79, 2554 (1997).

${ }^{30}$ R. Moessner and J. T. Chalker, Phys. Rev. B 58, 12049 (1998).

${ }^{31}$ C. L. Henley, Phys. Rev. B 71, 014424 (2005).

${ }^{32}$ C. Castelnovo, R. Moessner, and S. L. Sondhi, Nature (London) 451, 42 (2008).

${ }^{33}$ J. P. Garrahan and M. E. J. Newman, Phys. Rev. E 62, 7670 (2000).

${ }^{34}$ K. G. Wilson, Phys. Rev. D 3, 1818 (1971).

${ }^{35}$ M. B. Hastings and X.-G. Wen, Phys. Rev. B 72, 045141 (2005).

${ }^{36}$ B. Yoshida, Ann. Phys. (NY) 326, 15 (2011).

${ }^{37}$ F. J. MacWilliams and N. J. A. Sloane, The Theory of ErrorCorrecting Codes (North-Holland, Amsterdam, 1977).

${ }^{38}$ B. Yoshida and I. L. Chuang, Phys. Rev. A 81, 052302 (2010).

${ }^{39}$ M. A. Nielsen and I. L. Chuang, Quantum Computation and Quantum Information (Cambridge University Press, Cambridge, 2000).

${ }^{40}$ S. Bravyi and J. Haah, Phys. Rev. Lett. 107, 150504 (2011).

${ }^{41}$ G. Vidal, Phys. Rev. Lett. 99, 220405 (2007).

${ }^{42} \mathrm{~F}$. Verstraete and J. I. Cirac, arXiv:cond-mat/0407066.

${ }^{43}$ Z.-C. Gu, M. Levin, and X.-G. Wen, Phys. Rev. B 78, 205116 (2008).

${ }^{44}$ G. Evenbly and G. Vidal, Phys. Rev. Lett. 102, 180406 (2009).

${ }^{45}$ S. A. Ocko and B. Yoshida, Phys. Rev. Lett. 107, 250502 (2011).

${ }^{46}$ S. Bravyi, D. Poulin, and B. Terhal, Phys. Rev. Lett. 104, 050503 (2010). 